\title{
Bi-relative algebraic $K$-theory and topological cyclic homology
}

\author{
Thomas Geisser and Lars Hesselholt
}

\section{Introduction}

It is well-known that algebraic $K$-theory preserves products of rings. However, in general, algebraic $K$-theory does not preserve fiber products of rings, and one defines bi-relative algebraic $K$-theory so as to measure the deviation. It was proved recently by Cortiñas [6] that, rationally, bi-relative algebraic $K$-theory and bi-relative negative cyclic homology agree. In this paper, we show that, with finite coefficients, bi-relative algebraic $K$-theory and bi-relative topological cyclic homology agree. As an application, we show that for a possibly singular curve over a perfect field $k$ of positive characteristic $p$, the cyclotomic trace map induces an isomorphism of the $p$-adic algebraic $K$-groups and the $p$-adic topological cyclic homology groups in non-negative degrees. As a further application, we show that the difference between the $p$-adic $K$-groups of the integral group ring of a finite group and the $p$-adic $K$-groups of a maximal $\mathbb{Z}$-order in the rational group algebra can be expressed entirely in terms of topological cyclic homology.

Let $F$ be a functor that to a unital associative ring $A$ associates a symmetric spectrum $F(A)$. If $I \subset A$ is a two-sided ideal, the relative term $F(A, I)$ is defined to be the homotopy fiber of the map $F(A) \rightarrow F(A / I)$ induced from the canonical projection. If further $f: A \rightarrow B$ is a ring homomorphism such that $f: I \rightarrow f(I)$ is an isomorphism onto a two-sided ideal of $B$, there is an induced map $F(A, I) \rightarrow$ $F(B, f(I))$, and the bi-relative term $F(A, B, I)$ is defined to be the homotopy fiber of this map. Then there is a distinguished triangle in the stable homotopy category

$$
F(A, B, I) \rightarrow F(A, I) \stackrel{f_{*}}{\rightarrow} F(B, f(I)) \stackrel{\partial}{\rightarrow} \Sigma F(A, B, I)
$$

which is natural with respect to the triple $(A, B, I)$. We write the induced longexact sequence of homotopy groups as

$$
\cdots \rightarrow F_{q}(A, B, I) \rightarrow F_{q}(A, I) \stackrel{f_{*}}{\rightarrow} F_{q}(B, f(I)) \stackrel{\partial}{\rightarrow} F_{q-1}(A, B, I) \rightarrow \cdots
$$

The theorem of Cortiñas [6] states that the trace map

$$
K_{q}(A) \otimes \mathbb{Q} \rightarrow \mathrm{HC}_{q}^{-}(A \otimes \mathbb{Q})
$$

Both authors were supported in part by the National Science Foundation (USA). The second named author received additional support from the COE (Japan). 
from rational $K$-theory of $A$ to negative cyclic homology of $A \otimes \mathbb{Q}$ induces an isomorphism of the associated bi-relative theories. In this paper we prove the following analogous result for the $K$-theory with finite coefficients:

TheOREm 1. Let $f: A \rightarrow B$ be a map of unital associative rings, let $I \subset A$ be a two-sided ideal and assume that $f: I \rightarrow f(I)$ is an isomorphism onto a two-sided ideal of $B$. Then the map induced by the cyclotomic trace map

$$
K_{q}\left(A, B, I, \mathbb{Z} / p^{v}\right) \rightarrow\left\{\mathrm{TC}_{q}^{n}\left(A, B, I ; p, \mathbb{Z} / p^{v}\right)\right\}_{n \geqslant 1}
$$

is an isomorphism of pro-abelian groups, for all integers $q$, all primes $p$, and all positive integers $v$.

We recall from Bass [1 Thm. XII.8.3] that the common pro-abelian group is zero, for $q \leqslant 0$. We also remark that Thm. 1 implies the slightly weaker statement that the map of spectra induced by the cyclotomic trace map

$$
K(A, B, I) \rightarrow \mathrm{TC}(A, B, I ; p)=\underset{n}{\operatorname{holim}} \mathrm{TC}^{n}(A, B, I ; p)
$$

becomes a weak equivalence after $p$-completion. However, for the proof, it is essential that we work with the pro-abelian groups and not pass to the limit. We also mention that Dundas and Kittang [9] have shown that Thm. 1] implies the analogous statement for connective symmetric ring spectra.

A weaker version of the theorem of Cortiñas was first stated as a conjecture by Geller, Reid, and Weibel [12, 13, and used to evaluate the rational algebraic $K$-theory of curves over a field of characteristic zero. In a similar manner, we obtain the following result from Thm. 1

TheOREM 2. Let $X \rightarrow$ Spec $k$ be any curve over a field $k$ of positive characteristic $p$. Then, for all positive integers $v$, the cyclotomic trace map

$$
K_{q}\left(X, \mathbb{Z} / p^{v}\right) \rightarrow\left\{\mathrm{TC}_{q}^{n}\left(X ; p, \mathbb{Z} / p^{v}\right)\right\}_{n \geqslant 1}
$$

is an isomorphism of pro-abelian groups in degrees $q \geqslant r$, where $p^{r}=\left[k: k^{p}\right]$ is the degree of $k$ over the subfield $k^{p}$ of pth powers.

In the proof of Thm. 2] which is given in Sect. 3 below, we use Thm. 10 to reduce to the smooth case which we have proved earlier [10 Thm. 4.2.2]. We remark again that Thm. 2 implies the slightly weaker statement that the map of spectra induced by the cyclotomic trace map

$$
K(X) \rightarrow \mathrm{TC}(X ; p)=\underset{n}{\operatorname{holim}} \operatorname{TC}^{n}(X ; p)
$$

becomes a weak equivalence after $p$-completion and after passage to $(r-1)$-connected covers. The topological cyclic homology groups on the right-hand side of the statement of Thm. 2] can often be effectively calculated. As an example, we have the following result; compare [12. The proof is similar to the proof of [16 Thm. E] and 17. Thm. A] and will appear in [14.

TheOREM 3. Let $k$ be a regular $\mathbb{F}_{p}$-algebra, let $A=k[x, y] /(x y)$ be the coordinate ring of the coordinate axes in the affine $k$-plane, and let $I \subset A$ be the ideal generated by the variables $x$ and $y$. Then for all positive integers $q$, there is a canonical isomorphism

$$
K_{q}(A, I) \stackrel{\sim}{\leftarrow} \bigoplus_{m \geqslant 1} \mathbf{W}_{m} \Omega_{k}^{q-2 m}
$$


where $\mathbf{W}_{m} \Omega_{k}^{j}$ is the group of big de Rham-Witt $j$-forms of $k$ [17, 15.

The group $K_{2}(A, I)$ was evaluated twenty-five years ago by Dennis and Krusemeyer [8], but it was previously only known that the higher relative $K$-groups are $p$-primary torsion groups [28. We point out that, at present, the structure of the $K$-groups of singular schemes is very far from understood. In particular, there is currently no motivic cohomology theory that explain the structure of the $K$-groups that we find here. To illustrate this, let $k$ be a perfect field of positive characteristic $p$. Then Thm. 3 shows that the $p$-adic $K$-groups of $X=\operatorname{Spec} k[x, y] /(x y)$ are concentrated in even degrees with the group in positive degree $2 m$ canonically isomorphic to the group $\mathbf{W}_{m}(k)$ of big Witt vectors of length $m$ in $k$. In particular, the $p$-adic $K$-groups of $X$ cannot be generated from $K_{1}(X)$ by means of products and transfers as is the case for smooth schemes.

Finally, let $A=\mathbb{Z}[G]$ be the integral group ring of a finite group, and let $B=\mathfrak{M}$ be a maximal $\mathbb{Z}$-order of the rational group algebra. Then Thm. 1 shows that, for every prime $p$, the difference between the $p$-adic $K$-groups of $A$ and $B$ can be expressed entirely in terms of topological cyclic homology. Indeed, if we choose $B$ such that $A \subset B$, then the ideal $I=|G| \cdot B$ is a common ideal of both $A$ and $B$, and the quotient rings $A / I$ and $B / I$ are finite. The precise relationship is most clearly expressed for $G$ a finite $p$-group where we obtain the following result. We are grateful to Holger Reich for drawing our attention to this application of Thm. 1

TheOREM 4. Let $A=\mathbb{Z}[G]$ be the integral group ring of a finite p-group, and let $B=\mathfrak{M}$ be a maximal $\mathbb{Z}$-order of the rational group algebra such that $A \subset B$. Then there is a natural sequence of pro-abelian groups

$$
\cdots \rightarrow K_{q}\left(A, \mathbb{Z} / p^{v}\right) \rightarrow\left\{\begin{array}{c}
K_{q}\left(B, \mathbb{Z} / p^{v}\right) \\
\oplus \\
\mathrm{TC}_{q}^{n}\left(A ; p, \mathbb{Z} / p^{v}\right)
\end{array}\right\} \rightarrow\left\{\mathrm{TC}_{q}^{n}\left(B ; p, \mathbb{Z} / p^{v}\right)\right\} \rightarrow \cdots
$$

which is exact, for all non-negative integers $q$, and all positive integers $v$.

We again remark that Thm. 4 implies the slightly weaker result that the following square of spectra becomes homotopy-cartesian after $p$-completion and passage to connective covers:

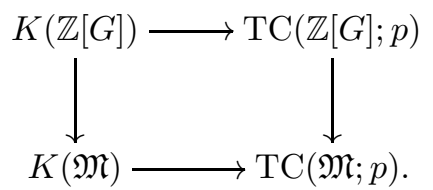

The strength of Thm. 目lies in the fact that the maximal order $\mathfrak{M}$ is a regular ring whose $K$-groups fit in the following localization sequence [22, Thm. 1.17]:

$$
\cdots \rightarrow \bigoplus_{\ell} K_{q}\left(\mathfrak{M}_{\ell} / J_{\ell}\right) \rightarrow K_{q}(\mathfrak{M}) \rightarrow K_{q}(\mathbb{Q}[G]) \rightarrow \cdots .
$$

The sum on the left-hand side ranges over all rational primes $\ell$ and $J_{\ell} \subset \mathfrak{M}_{\ell}$ is the Jacobson radical in the $\ell$-adic completion of $\mathfrak{M}$. The rings $\mathbb{Q}[G]$ and $\mathfrak{M}_{\ell} / J_{\ell}$ are semi-simple.

The proof of Thm. 1 is similar, in outline, to the proof of the theorem of Cortiñas [6] and uses two key observations by Cuntz and Quillen [7. The first observation is that it suffices to prove Thm. 1 in the case where the non-unital associative ring $I$ can be embedded as a two-sided ideal of a free unital associative 
ring. The second observation is that, in this case, the pro-algebra $\left\{I^{m}\right\}$ is homological unital in the sense that for all primes $p$, and all positive integers $q$, the following pro-abelian group is zero:

$$
\left\{\operatorname{Tor}_{q}^{\mathbb{Z} \ltimes I^{m}}(\mathbb{Z}, \mathbb{Z} / p)\right\}_{m \geqslant 1} .
$$

Here $\mathbb{Z} \ltimes I^{m}$ is the unital associative ring obtained from $I^{m}$ by adjoining a unit. In Sect. 1 below we show that this implies that for all integers $q$, all primes $p$, and all positive integers $v$, the pro-abelian group

$$
\left\{K_{q}\left(A, B, I^{m}, \mathbb{Z} / p^{v}\right)\right\}_{m \geqslant 1}
$$

is zero. This result is a generalization of the excision theorem of Suslin-Wodzicki 25, 26. Similarly, we show in Sect. 2 that for all integers $q$, all primes $p$, and all positive integers $v$, the pro-abelian group

$$
\left\{\mathrm{TC}_{q}^{n}\left(A, B, I^{m} ; p, \mathbb{Z} / p^{v}\right)\right\}_{m, n \geqslant 1}
$$

is zero. Hence, if $I$ can be embedded as an ideal of a free unital associative ring, the cyclotomic trace map induces an isomorphism of pro-abelian groups in the top row of the following diagram:

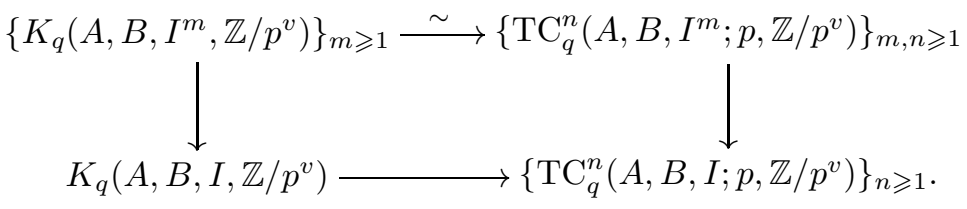

Finally, it follows from a theorem of McCarthy [21 and from [1] Thm. 2.2.1] that also the lower horizontal map is an isomorphism of pro-abelian groups. The details of this argument are given in Sect. 3 below. This completes the outline of the proof of Thm. 1]

A pro-object of a category $\mathcal{C}$ is a functor from a directed partially ordered set to the category $\mathcal{C}$, and a strict map between two pro-objects with the same indexing set is a natural transformation. A general map from a pro-object $X=\left\{X_{i}\right\}_{i \in I}$ to a pro-object $Y=\left\{Y_{j}\right\}_{j \in J}$ is an element of the set

$$
\operatorname{Hom}_{\text {pro }-\mathcal{C}}(X, Y)=\lim _{J} \operatorname{colim}_{I} \operatorname{Hom}_{\mathcal{C}}\left(X_{i}, Y_{j}\right) \text {. }
$$

In particular, a pro-object $X=\left\{X_{i}\right\}_{i \in I}$ in a category with a null-object is zero if for all $i \in I$, there exists $i^{\prime} \geqslant i$ such that the map $X_{i^{\prime}} \rightarrow X_{i}$ is zero. We write $X[-]$ for a simplicial object in a category $\mathcal{C}$, and we write $X[k]$ for the object in simplicial degree $k$. If $I$ is a non-unital associative ring, we write $\mathbb{Z} \ltimes I$ for the unital associative ring given by the product $\mathbb{Z} \times I$ with multiplication $(n, x) \cdot\left(n^{\prime}, x^{\prime}\right)=$ $\left(n n^{\prime}, n x^{\prime}+n^{\prime} x+x x^{\prime}\right)$.

This paper was written in part while the authors visited the University of Tokyo. We would like to express our sincere gratitude to the university and to Takeshi Saito for their kind hospitality and for the stimulating atmosphere they provided. Finally, it is a particular pleasure to acknowledge the help we have received from Guillermo Cortiñas. He long ago suggested to us to prove the main theorem of this paper by adapting the integral excision theorem of Suslin [25] to the pro-setting. He also pointed out a gap in our arguments in an earlier version of this paper. 


\section{1. $K$-theory}

In this section we prove the following variant of the excision theorem of SuslinWodzicki [26, 25]. Our treatment closely follows Suslin [25].

Theorem 1.1. Let $f: A \rightarrow B$ be a map of unital associative rings, and let $I \subset A$ be a two-sided ideal such that $f: I \rightarrow f(I)$ is an isomorphism onto a twosided ideal of $B$. Let $p$ be a prime, and suppose that for all positive integers $q$, the pro-abelian group

$$
\left\{\operatorname{Tor}_{q}^{\mathbb{Z} \ltimes I^{m}}(\mathbb{Z}, \mathbb{Z} / p)\right\}_{m \geqslant 1}
$$

is zero. Then for all integers $q$, and all positive integers $v$, the map of pro-abelian groups

is an isomorphism.

$$
\left\{K_{q}\left(A, I^{m}, \mathbb{Z} / p^{v}\right)\right\}_{m \geqslant 1} \rightarrow\left\{K_{q}\left(B, f(I)^{m}, \mathbb{Z} / p^{v}\right)\right\}_{m \geqslant 1}
$$

We will use a functorial version of the plus-construction of Quillen. So

$$
\lambda: X \rightarrow X^{+}
$$

will be a natural transformation of functors from the category of pointed spaces and base-point preserving continuous maps to itself. For example, one can use the functorial localization [19 Def. 1.2.2] with respect to a pointed space $W$ whose reduced integral homology is trivial and whose fundamental group admits a nontrivial group homomorphism to $G L(\mathbb{Z})[\mathbf{2}$.

Let $A$ be a unital associative ring, and let $I \subset A$ be a two-sided ideal. Let $G L(I)$ and $\overline{G L}(A / I)$ be the kernel and image of the map $G L(A) \rightarrow G L(A / I)$ induced by the canonical projection. We let $F(A, I)$ be the homotopy fiber of the canonical map $B G L(A)^{+} \rightarrow B \overline{G L}(A / I)^{+}$, and let $X(A, I)$ be the homotopy pullback of $B \overline{G L}(A / I)$ and $B G L(A)^{+}$over $B \overline{G L}(A / I)^{+}$. We then have the following diagram of pointed spaces and base-point preserving continuous maps where the rows are fiber sequences:

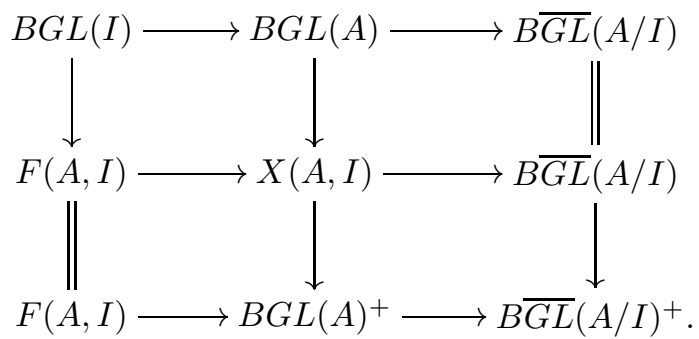

We consider the relative Serre spectral sequence

$$
\begin{aligned}
E_{s, t}^{2} & =H_{s}\left(\overline{G L}(A / I), H_{t}(F(A, I), B G L(I), \mathbb{Z} / p)\right) \\
& \Rightarrow H_{s+t}(X(A, I), B G L(A), \mathbb{Z} / p)
\end{aligned}
$$

which converges to zero. Indeed, both of the maps $B G L(A) \rightarrow B G L(A)^{+}$and $X(A, I) \rightarrow B G L(A)^{+}$induce isomorphisms on homology, and hence so does the map $B G L(A) \rightarrow X(A, I)$. Hence, the relative homology groups, defined as the reduced homology groups of the mapping cone, are zero. The diagram and the spectral sequence considered above both are functorial in the pair $I \subset A$. Hence, if we consider the pro-ideal $\left\{I^{m}\right\}_{m \geqslant 1}$ of powers of the ideal $I \subset A$, we obtain a spectral sequence of pro-abelian groups. 
LEMMA 1.2. If the pro-abelian group $\left\{H_{t}\left(F\left(A, I^{m}\right), B G L\left(I^{m}\right), \mathbb{Z} / p\right)\right\}$ is zero, for $0 \leqslant t<q$, then the pro-abelian group

$$
\left\{H_{s}\left(\overline{G L}\left(A / I^{m}\right), H_{q}\left(F\left(A, I^{m}\right), B G L\left(I^{m}\right)\right), \mathbb{Z} / p\right)\right\}
$$

is zero, for $0 \leqslant s \leqslant 1$. If, in addition, the canonical map

$$
\left\{H_{q-1}\left(B G L\left(I^{m}\right), \mathbb{Z} / p\right)\right\} \rightarrow\left\{H_{0}\left(\overline{G L}\left(A / I^{m}\right), H_{q-1}\left(B G L\left(I^{m}\right), \mathbb{Z} / p\right)\right)\right\}
$$

is an isomorphism of pro-abelian groups, then the pro-abelian group

$$
\left\{H_{q}\left(F\left(A, I^{m}\right), B G L\left(I^{m}\right), \mathbb{Z} / p\right)\right\}
$$

is zero.

ProOF. We omit the $\mathbb{Z} / p$-coefficients for the homology throughout the proof. The first claim follows immediately from the spectral sequence. To prove the second claim, we show that in the following exact sequence of pro-abelian groups both maps are zero:

$$
\left\{H_{q}\left(F\left(A, I^{m}\right)\right)\right\} \stackrel{j}{\rightarrow}\left\{H_{q}\left(F\left(A, I^{m}\right), B G L\left(I^{m}\right)\right)\right\} \stackrel{\partial}{\rightarrow}\left\{H_{q-1}\left(B G L\left(I^{m}\right)\right)\right\} .
$$

We first prove that the map $\partial$ is zero, or equivalently, that in the following exact sequence of pro-abelian groups, the map $i$ is injective:

$$
\left\{H_{q}\left(F\left(A, I^{m}\right), B G L\left(I^{m}\right)\right)\right\} \stackrel{\partial}{\rightarrow}\left\{H_{q-1}\left(B G L\left(I^{m}\right)\right)\right\} \stackrel{i}{\rightarrow}\left\{H_{q-1}\left(F\left(A, I^{m}\right)\right)\right\} .
$$

By hypothesis, the map $i$ is surjective, and therefore, the induced sequence of $\left\{\overline{G L}\left(A / I^{m}\right)\right\}$-coinvariants again is exact. Hence, the first statement of the lemma shows that $\partial$ induces an monomorphism of pro-abelian groups

$$
\left\{H_{0}\left(\overline{G L}\left(A / I^{m}\right), H_{q-1}\left(B G L\left(I^{m}\right)\right)\right)\right\} \stackrel{\bar{\partial}}{\rightarrow}\left\{H_{0}\left(\overline{G L}\left(A / I^{m}\right), H_{q-1}\left(F\left(A, I^{m}\right)\right)\right)\right\} .
$$

But, by further hypothesis, the canonical projection

$$
\left\{H_{q-1}\left(B G L\left(I^{m}\right)\right)\right\} \stackrel{\pi^{\prime}}{\rightarrow}\left\{H_{0}\left(\overline{G L}\left(A / I^{m}\right), H_{q-1}\left(B G L\left(I^{m}\right)\right)\right)\right\}
$$

is an isomorphism, and hence, the map $\partial$ is injective as desired.

It remains to show that the map $j$ is zero, or equivalently, that in the following exact sequence of pro-abelian groups, the $i$ map is surjective:

$$
\left\{H_{q}\left(B G L\left(I^{m}\right)\right)\right\} \stackrel{i}{\rightarrow}\left\{H_{q}\left(F\left(A, I^{m}\right)\right)\right\} \stackrel{j}{\rightarrow}\left\{H_{q}\left(F\left(A, I^{m}\right), B G L\left(I^{m}\right)\right)\right\} .
$$

The map $i$ is surjective by what was proved above, so the induced sequence of $\left\{\overline{G L}\left(A / I^{m}\right)\right\}$-coinvariants again is exact. Therefore, by the first statement of the lemma, the induced map

$$
\left\{H_{0}\left(\overline{G L}\left(A / I^{m}\right), H_{q}\left(B G L\left(I^{m}\right)\right)\right)\right\} \stackrel{\bar{i}}{\rightarrow}\left\{H_{0}\left(\overline{G L}\left(A / I^{m}\right), H_{q}\left(F\left(A, I^{m}\right)\right)\right)\right\}
$$

is surjective, so it suffices to show that the canonical projection

$$
\left\{H_{q}\left(F\left(A, I^{m}\right)\right\} \stackrel{\pi}{\rightarrow}\left\{H_{0}\left(\overline{G L}\left(A / I^{m}\right), H_{q}\left(F\left(A, I^{m}\right)\right)\right)\right\}\right.
$$

is an isomorphism. But $X\left(A, I^{m}\right)$ is a homotopy pull-back, and hence, the action of $\overline{G L}\left(A / I^{m}\right)=\pi_{1}\left(B \overline{G L}\left(A / I^{m}\right)\right)$ on $H_{q}\left(F\left(A, I^{m}\right)\right)$ is induced from the action of $\pi_{1}\left(B \overline{G L}\left(A / I^{m}\right)^{+}\right)$on $H_{q}\left(F\left(A, I^{m}\right)\right)$. Finally,

$$
F\left(A, I^{m}\right) \rightarrow B G L(A)^{+} \rightarrow B \overline{G L}\left(A / I^{m}\right)^{+}
$$

is a fiber sequence of infinite loop spaces, so the fundamental group of the base acts trivially on the homology of the fiber. 
Proposition 1.3. Let $A$ be a unital associative ring, and let $I \subset A$ be a twosided ideal. Let $p$ be a prime, and assume that the pro-abelian group

$$
\left\{\operatorname{Tor}_{q}^{\mathbb{Z} \ltimes I^{m}}(\mathbb{Z}, \mathbb{Z} / p)\right\}_{m \geqslant 1}
$$

is zero, for all positive integers $q$. Then the pro-abelian group

$$
\left\{H_{q}\left(F\left(A, I^{m}\right), B G L\left(I^{m}\right), \mathbb{Z} / p\right)\right\}_{m \geqslant 1}
$$

is zero, for all integers $q$.

Proof. We omit the $\mathbb{Z} / p$-coefficients for the homology. We show that the canonical projection induces an isomorphism of pro-abelian groups

$$
\left\{H_{q}\left(B G L\left(I^{m}\right)\right)\right\} \stackrel{\sim}{\longrightarrow}\left\{H_{0}\left(\overline{G L}\left(A / I^{m}\right), H_{q}\left(B G L\left(I^{m}\right)\right)\right)\right\},
$$

for all integers $q$. The proposition then follows from Lemma 1.2 by induction on $q$ starting from the trivial case $q=0$.

It suffices, by the proof of [26 Cor. 1.6], to show that for all $m \geqslant 1$, there exists $k \geqslant m$ such that the image of the map

$$
\iota_{k, m *}: H_{q}\left(G L\left(I^{k}\right)\right) \rightarrow H_{q}\left(G L\left(I^{m}\right)\right)
$$

is invariant under the adjoint action by $G L(\mathbb{Z})$. And by op. cit., Lemma 1.4, the embedding $\varphi: G L\left(I^{m}\right) \rightarrow G L\left(I^{m}\right)$ given by

$$
\varphi(\alpha)=\left(\begin{array}{ll}
1 & 0 \\
0 & \alpha
\end{array}\right)
$$

induces an injection on homology, so it suffices to show that the image of the composite map

$$
H_{q}\left(G L\left(I^{k}\right)\right) \stackrel{\iota_{k, m *}}{\longrightarrow} H_{q}\left(G L\left(I^{m}\right)\right) \stackrel{\varphi_{*}}{\longrightarrow} H_{q}\left(G L\left(I^{m}\right)\right)
$$

is invariant under the adjoint action by the subgroup $\varphi(G L(\mathbb{Z})) \subset G L(\mathbb{Z})$. In effect, we show that the image of $\varphi_{*} \circ \iota_{k, m *}$ is invariant under the adjoint action by the full group $G L(\mathbb{Z})$. We recall that $G L(\mathbb{Z})$ is generated by the diagonal matrix $\operatorname{diag}(-1,1,1, \ldots)$ and by the elementary matrices $e_{s, 1}(1)$ and $e_{1, s}(1)$, where $s>1$. It is clear that the image of $\varphi_{*} \circ \iota_{k, m *}$ is invariant under the action of the diagonal matrix $\operatorname{diag}(-1,1,1, \ldots)$. To show that the image of $\varphi_{*} \circ \iota_{k, m *}$ is invariant under the action of the elementary matrices $e_{s, 1}(1)$ we consider the semi-direct product group

$$
\Gamma\left(I^{m}\right)=G L\left(I^{m}\right) \ltimes M_{\infty, 1}\left(I^{m}\right),
$$

where $G L\left(I^{m}\right)$ acts on the group $M_{\infty, 1}\left(I^{m}\right)$ of column vectors by left multiplication. Let $\sigma: G L\left(I^{m}\right) \rightarrow \Gamma\left(I^{m}\right)$ and $\pi: \Gamma\left(I^{m}\right) \rightarrow G L\left(I^{m}\right)$ be the canonical group homomorphisms given by $\sigma(\alpha)=(\alpha, 0)$ and $\pi(\alpha, v)=\alpha$. Then the map $\varphi$ is equal to the composition of the group homomorphisms

$$
G L\left(I^{m}\right) \stackrel{\sigma}{\rightarrow} \Gamma\left(I^{m}\right) \stackrel{j}{\rightarrow} G L\left(I^{m}\right),
$$

where

$$
j(\alpha, v)=\left(\begin{array}{ll}
1 & 0 \\
v & \alpha
\end{array}\right) .
$$

The matrix $e_{s, 1}(1) \in G L(\mathbb{Z})$ is the image by $j: \Gamma(\mathbb{Z}) \rightarrow G L(\mathbb{Z})$ of a unique matrix $e_{s, 1}^{\prime}(1) \in \Gamma(\mathbb{Z})$ and $\pi\left(e_{s, 1}^{\prime}(1)\right) \in G L(\mathbb{Z})$ is the identity matrix. It suffices to show that there exists $k \geqslant m$ such that the image of the map

$$
\iota_{k, m *}: H_{q}\left(\Gamma\left(I^{k}\right)\right) \rightarrow H_{q}\left(\Gamma\left(I^{m}\right)\right)
$$


is invariant under the adjoint action of the matrices $e_{s, 1}^{\prime}(1), s>1$. It follows from Prop. 1.9 below that there exists $k \geqslant m$ such that

$$
\iota_{k, m *}=\iota_{k, m *} \circ \sigma_{*} \circ \pi_{*}: H_{q}\left(\Gamma\left(I^{k}\right)\right) \rightarrow H_{q}\left(\Gamma\left(I^{m}\right)\right) .
$$

Granting this, we obtain that

$$
\begin{aligned}
\operatorname{Ad}\left(e_{s, 1}^{\prime}(1)\right)_{*} \circ \iota_{k, m *} & =\iota_{k, m *} \circ \operatorname{Ad}\left(e_{s, 1}^{\prime}(1)\right)_{*} \\
& =\iota_{k, m *} \circ \sigma_{*} \circ \pi_{*} \circ \operatorname{Ad}\left(e_{s, 1}^{\prime}(1)\right)_{*} \\
& =\iota_{k, m *} \circ \sigma_{*} \circ \operatorname{Ad}\left(\pi\left(e_{s, 1}^{\prime}(1)\right)\right)_{*} \circ \pi_{*} \\
& =\iota_{k, m *} \circ \sigma_{*} \circ \pi_{*}=\iota_{k, m *}
\end{aligned}
$$

as desired. An analogous argument shows that there exists (a possibly larger) $k \geqslant m$ such that the image of $\varphi_{*} \circ \iota_{k, m *}$ is invariant under the action of the elementary matrices $e_{1, s}(1)$.

Proof of Thm. 1.1. It suffices by simple induction to treat the case $v=1$. Let $f: A \rightarrow B$ be as in the statement. By Prop. 1.3 the induced map

$$
\left\{H_{q}\left(F\left(A, I^{m}\right), \mathbb{Z} / p\right)\right\}_{m \geqslant 1} \rightarrow\left\{H_{q}\left(F\left(B, f(I)^{m}\right), \mathbb{Z} / p\right)\right\}_{m \geqslant 1}
$$

is an isomorphism of pro-abelian groups, and [23, Cor. 5.8] then shows that also the induced map

$$
\left\{\pi_{q}\left(F\left(A, I^{m}\right), \mathbb{Z} / p\right)\right\}_{m \geqslant 1} \rightarrow\left\{\pi_{q}\left(F\left(B, f(I)^{m}\right), \mathbb{Z} / p\right)\right\}_{m \geqslant 1}
$$

is an isomorphism of pro-abelian groups.

The remainder of this section is devoted to the proof of Prop. 1.9 below which we used in the proof of Prop. 1.3 above. We begin with some general theory.

Let $A$ be a unital associative ring, and let $I \subset A$ be a two-sided ideal. Then the powers $\left\{I^{m}\right\}_{m \geqslant 1}$ form a pro-associative ring without unit. We say that a proabelian group $\left\{M_{m}\right\}_{m \geqslant 1}$ is a left module over $\left\{I^{m}\right\}_{m \geqslant 1}$ if for all $m \geqslant 1, M_{m}$ is a left $I^{m}$-module and if for all $k \geqslant m \geqslant 1$, the following diagram commutes:

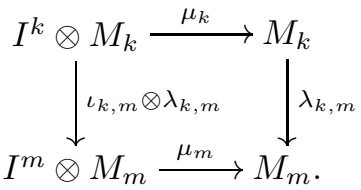

By a homomorphism from a left $\left\{I^{m}\right\}$-module $\left\{M_{m}\right\}$ to a left $\left\{I^{m}\right\}$-module $\left\{M_{m}^{\prime}\right\}$, we mean a strict map of pro-abelian groups $f:\left\{M_{m}\right\} \rightarrow\left\{M_{m}^{\prime}\right\}$ such that for all $m \geqslant 1$, the map $f_{m}: M_{m} \rightarrow M_{m}^{\prime}$ is an $I^{m}$-module homomorphism. We say that a left $\left\{I^{m}\right\}$-module $\left\{P_{m}\right\}$ is pseudo-free if for all $m \geqslant 1$, there are $I^{m}$-module isomorphisms $\varphi_{m}: I^{m} \otimes L_{m}$ xrightarrow $\sim P_{m}$ with $L_{m}$ a free abelian group, and if for all $k \geqslant m \geqslant 1$, there are maps of abelian groups $\sigma_{k, m}: L_{k} \rightarrow L_{m}$ such that the following diagrams commute:

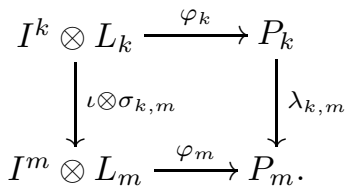

A homomorphism $f:\left\{P_{m}\right\} \rightarrow\left\{M_{m}\right\}$ from a pseudo-free left $\left\{I^{m}\right\}$-module to an arbitrary left $\left\{I^{m}\right\}$-module is said to be 
(i) a special homomorphism if there exists a strict map of pro-abelian groups $g:\left\{L_{m}\right\} \rightarrow\left\{M_{m}\right\}$ such that for all $m \geqslant 1$, the composite

$$
I^{m} \otimes L_{m} \stackrel{\varphi_{m}}{\longrightarrow} P_{m} \stackrel{f_{m}}{\longrightarrow} M_{m}
$$

maps $a \otimes x$ to $a g_{m}(x)$.

(ii) a $p^{v}$-special homomorphism if the left $\left\{I^{m}\right\}$-module $\left\{P_{m}\right\}$ admits a strict direct sum decomposition

$$
\iota^{\prime} \oplus \iota^{\prime \prime}:\left\{P_{m}^{\prime}\right\} \oplus\left\{P_{m}^{\prime \prime}\right\} \stackrel{\sim}{\longrightarrow}\left\{P_{m}\right\}
$$

such that $\left\{P_{m}^{\prime}\right\}$ and $\left\{P_{m}^{\prime \prime}\right\}$ are pseudo-free, such that $f \circ \iota^{\prime}$ is special, and such that $f \circ \iota^{\prime \prime}=p^{v} g$, for some homomorphism $g:\left\{P_{m}^{\prime \prime}\right\} \rightarrow\left\{M_{m}\right\}$.

Lemma 1.4. Let $\left\{M_{m}\right\}$ be a left $\left\{I^{m}\right\}$-module. Then there exists a special homomorphism $f:\left\{P_{m}\right\} \rightarrow\left\{M_{m}\right\}$ from a pseudo-free left $\left\{I^{m}\right\}$-module $\left\{P_{m}\right\}$ such that the image of $f_{m}$ is equal to $I^{m} \cdot M_{m} \subset M_{m}$.

Proof. Let $\mathbb{Z}\left\{M_{m}\right\}$ be the free abelian group generated by the set of elements of $M_{m}$. We define $P_{m}$ to be the extended left $I^{m}$-module

$$
P_{m}=I^{m} \otimes \bigoplus_{s \geqslant m} \mathbb{Z}\left\{M_{s}\right\}
$$

and $\lambda_{k, m}: P_{k} \rightarrow P_{m}$ to be the tensor product of the canonical inclusions. It is clear that $\left\{P_{m}\right\}$ is a pseudo-free left $\left\{I^{m}\right\}$-module and that the canonical map $f:\left\{P_{m}\right\} \rightarrow\left\{M_{m}\right\}$ is a special homomorphism such that the image of $f_{m}$ is equal to $I^{m} \cdot M_{m} \subset M_{m}$ as desired.

Let $\left\{P_{m}\right\}$ be a pseudo-free left $\left\{I^{m}\right\}$-module. By an augmented complex of left $\left\{I^{m}\right\}$-modules over $\left\{P_{m}\right\}$, we mean a sequence of left $\left\{I^{m}\right\}$-modules and homomorphisms

$$
\ldots \stackrel{d}{\rightarrow}\left\{C_{m, 2}\right\} \stackrel{d}{\rightarrow}\left\{C_{m, 1}\right\} \stackrel{d}{\rightarrow}\left\{C_{m, 0}\right\} \stackrel{\epsilon}{\rightarrow}\left\{P_{m}\right\}
$$

such that $d_{m} \circ d_{m}$ and $\epsilon_{m} \circ d_{m}$ are equal to zero, for all $m \geqslant 1$. We say that the augmented complex $\epsilon:\left\{C_{m, *}\right\} \rightarrow\left\{P_{m}\right\}$ is

(i) special if the $\left\{C_{m, q}\right\}$ are pseudo-free, if $\epsilon$ is special, and if the homology of the augmented complex $\epsilon_{m}: C_{m, *} \rightarrow P_{m}$ is annihilated by $I^{m}$.

(ii) $p^{v}$-special if the $\left\{C_{m, q}\right\}$ are pseudo-free, if $\epsilon$ is $p^{v}$-special, and if the homology of the augmented complex $\epsilon_{m}: C_{m, *} \rightarrow P_{m}$ is annihilated both by $I^{m}$ and by $p^{v}$.

We remark that, for a pseudo-free left $\left\{I^{m}\right\}$-module $\left\{P_{m}\right\}$, the identity homomorphism is usually not special, and hence, does not constitute an augmented special complex.

Corollary 1.5. Let $\left\{P_{m}\right\}$ be a pseudo-free left $\left\{I^{m}\right\}$-module. Then there exists both a special augmented complex and a $p^{v}$-special augmented complex over $\left\{P_{m}\right\}$.

PROOF. It is proved by an easy induction argument based on Lemma 1.4 that there exists a special augmented complex

$$
\cdots \stackrel{d}{\longrightarrow}\left\{C_{m, 2}\right\} \stackrel{d}{\longrightarrow}\left\{C_{m, 1}\right\} \stackrel{d}{\longrightarrow}\left\{C_{m, 0}\right\} \stackrel{\epsilon}{\longrightarrow}\left\{P_{m}\right\} .
$$


Then the total complex of the double-complex

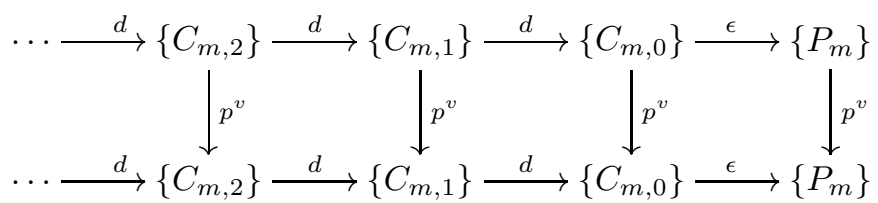

is a $p^{v}$-special augmented complex.

Proposition 1.6. Let $A$ be a unital associative ring, and let $I \subset A$ be a twosided ideal. Let $p$ be a prime, and assume that for every positive integer $q$, the following pro-abelian group is zero:

$$
\left\{\operatorname{Tor}_{q}^{\mathbb{Z} \ltimes I^{m}}(\mathbb{Z}, \mathbb{Z} / p)\right\}_{m \geqslant 1} .
$$

Then every $p^{v}$-special augmented complex of $\left\{I^{m}\right\}$-modules

$$
\ldots \stackrel{d}{\rightarrow}\left\{C_{m, 2}\right\} \stackrel{d}{\rightarrow}\left\{C_{m, 1}\right\} \stackrel{d}{\rightarrow}\left\{C_{m, 0}\right\} \stackrel{\epsilon}{\rightarrow}\left\{P_{m}\right\},
$$

is exact as an augmented complex of pro-abelian groups.

Proof. It follows by simple induction that the pro-abelian group

$$
\left\{\operatorname{Tor}_{q}^{\mathbb{Z} \ltimes I^{m}}\left(\mathbb{Z}, \mathbb{Z} / p^{v}\right)\right\}_{m \geqslant 1}
$$

is zero, for all positive integers $q$ and $v$. We show by induction on the degree that the following complex of pro-abelian groups has zero homology:

$$
\ldots \stackrel{d}{\rightarrow}\left\{C_{m, 2}\right\} \stackrel{d}{\rightarrow}\left\{C_{m, 1}\right\} \stackrel{d}{\rightarrow}\left\{C_{m, 0}\right\} \stackrel{d}{\rightarrow}\left\{C_{m,-1}\right\}=\left\{P_{m}\right\} .
$$

We let $Z_{m, q}$ and $B_{m, q-1}$ be the kernel and image of $d_{m}: C_{m, q} \rightarrow C_{m, q-1}$. Since $I^{m}$ and $p^{v}$ both annihilate $H_{q}\left(C_{m, *}\right)=Z_{m, q} / B_{m, q}$, we have

$$
I^{m} Z_{m, q}+p^{v} Z_{m, q} \subset B_{m, q} .
$$

We first consider $q=-1$. Then $Z_{m,-1}=P_{m}$, and hence $I^{m} P_{m}+p^{v} P_{m}$ is contained in $B_{m,-1}$. But the definition of a $p^{v}$-special homomorphism shows that also $B_{m,-1} \subset I^{m} P_{m}+p^{v} P_{m}$. It follows that we have an isomorphism

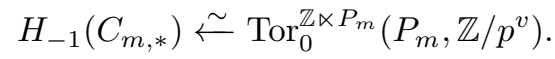

Now, since $\left\{P_{m}\right\}$ is a pseudo-free left $\left\{I^{m}\right\}$-module, we have an isomorphism of left $I^{m}$-modules $I^{m} \otimes L_{m} \stackrel{\sim}{\rightarrow} P_{m}$, with $L_{m}$ a free abelian group, so

$$
\begin{aligned}
H_{-1}\left(C_{m, *}\right) & \stackrel{\sim}{\sim} \operatorname{Tor}_{0}^{\mathbb{Z} \ltimes I^{m}}\left(P_{m}, \mathbb{Z} / p^{v}\right) \stackrel{\sim}{\leftarrow} \operatorname{Tor}_{0}^{\mathbb{Z} \ltimes I^{m}}\left(I^{m} \otimes L_{m}, \mathbb{Z} / p^{v}\right) \\
& \sim \operatorname{Tor}_{0}^{\mathbb{Z} \ltimes I^{m}}\left(I^{m}, \mathbb{Z} / p^{v}\right) \otimes L_{m} \stackrel{\sim}{\leftarrow} \operatorname{Tor}_{1}^{\mathbb{Z} \ltimes I^{m}}\left(\mathbb{Z}, \mathbb{Z} / p^{v}\right) \otimes L_{m} .
\end{aligned}
$$

This show that the pro-abelian group $\left\{H_{-1}\left(C_{m, *}\right)\right\}$ is zero as stated.

Next, we assume, inductively, that the pro-abelian group $\left\{H_{i}\left(C_{m, *}\right)\right\}$ is zero, for $i<q$, and show that the pro-abelian group $\left\{H_{q}\left(C_{m, *}\right)\right\}$ is zero. The inductive hypothesis is equivalent to the statement that the following sequence of pro-abelian groups is exact:

$$
0 \rightarrow\left\{Z_{m, q}\right\} \rightarrow\left\{C_{m, q}\right\} \rightarrow \cdots \rightarrow\left\{C_{m, 0}\right\} \rightarrow\left\{P_{m}\right\} \rightarrow 0 .
$$


This gives rise to the following spectral sequence of pro-abelian groups:

$$
\begin{aligned}
E_{s, t}^{1} & = \begin{cases}\left\{\operatorname{Tor}_{t}^{\mathbb{Z} \ltimes I^{m}}\left(C_{m, s}, \mathbb{Z} / p^{v}\right)\right\}, & \text { if } 0 \leqslant s \leqslant q, \\
\left\{\operatorname{Tor}_{t}^{\mathbb{Z} \ltimes I^{m}}\left(Z_{m, q}, \mathbb{Z} / p^{v}\right)\right\}, & \text { if } s=q+1, \\
\{0\}, & \text { if } s>q+1,\end{cases} \\
& \Rightarrow\left\{\operatorname{Tor}_{s+t}^{\mathbb{Z} \ltimes I^{m}}\left(P_{m}, \mathbb{Z} / p^{v}\right)\right\} .
\end{aligned}
$$

Since the left $\left\{I^{m}\right\}$-modules $\left\{C_{m, s}\right\}$ are pseudo-free, we see as before that, for $0 \leqslant$ $s \leqslant q$, the pro-abelian groups $E_{s, t}^{1}$ are zero. It follows that the edge-homomorphism of the spectral sequence

$$
\left\{\operatorname{Tor}_{q+1}^{\mathbb{Z} \ltimes I^{m}}\left(P_{m}, \mathbb{Z} / p^{v}\right)\right\} \rightarrow\left\{\operatorname{Tor}_{0}^{\mathbb{Z} \ltimes I^{m}}\left(Z_{m, q}, \mathbb{Z} / p^{v}\right)\right\}
$$

is an isomorphism of pro-abelian groups. The pro-abelian group on the left-hand side is zero, since the left $\left\{I^{m}\right\}$-module $\left\{P_{m}\right\}$ is pseudo-free. Hence, we may conclude that the following pro-abelian group is zero:

$$
\left\{\operatorname{Tor}_{0}^{\mathbb{Z} \ltimes I^{m}}\left(Z_{m, q}, \mathbb{Z} / p^{v}\right)\right\}=\left\{Z_{m, q} /\left(I^{m} Z_{m, q}+p^{v} Z_{m, q}\right)\right\} .
$$

Finally, since $I^{m} Z_{m, q}+p^{v} Z_{m, q}$ is contained in $B_{m, q}$, this pro-abelian group surjects onto the pro-abelian group $\left\{Z_{m, q} / B_{m, q}\right\}=\left\{H_{q}\left(C_{m, *}\right)\right\}$ which therefore is zero as stated.

Proposition 1.7. Let $A$ be a unital associative ring and let $I \subset A$ be a twosided ideal. Let $p$ be a prime and assume that for every positive integer $q$, the following pro-abelian group is zero:

$$
\left\{\operatorname{Tor}_{q}^{\mathbb{Z} \ltimes I^{m}}(\mathbb{Z}, \mathbb{Z} / p)\right\}_{m \geqslant 1} .
$$

Suppose that $F_{i}, i \geqslant 0$, is a sequence of possibly non-additive functors from the category of $\left\{I^{m}\right\}$-modules to the category of pro-abelian groups that satisfy the following conditions (i) - (iii):

(i) For every $\left\{I^{m}\right\}$-module $\left\{M_{m}\right\}$, the pro-abelian group $F_{0}\left(\left\{M_{m}\right\}\right)$ is zero.

(ii) If $\epsilon:\left\{M_{m}[-]\right\} \rightarrow\left\{M_{m}\right\}$ is an augmented simplicial $\left\{I^{m}\right\}$-module such that the associated augmented complex of pro-abelian groups is exact, then there is a spectral sequence of pro-abelian groups

$$
E_{s, t}^{1}=F_{t}\left(\left\{M_{m}[s]\right\}\right) \Rightarrow F_{s+t}\left(\left\{M_{m}\right\}\right)
$$

such that the edge-homomorphism $F_{t}\left(\left\{M_{m}[0]\right\}\right) \rightarrow F_{t}\left(\left\{M_{m}\right\}\right)$ is the map induced by the augmentation $\epsilon:\left\{M_{m}[0]\right\} \rightarrow\left\{M_{m}\right\}$.

(iii) For every $i \geqslant 0$, there exists $v \geqslant 1$ such that for every $p^{v}$-special homomorphism $f:\left\{P_{m}\right\} \rightarrow\left\{M_{m}\right\}$, the induced map of pro-abelian groups

$$
f_{*}: F_{i}\left(\left\{P_{m}\right\}\right) \rightarrow F_{i}\left(\left\{M_{m}\right\}\right)
$$

is zero.

Then for every pseudo-free $\left\{I^{m}\right\}$-module $\left\{P_{m}\right\}$ and for every $i \geqslant 0$, the proabelian group $F_{i}\left(\left\{P_{m}\right\}\right)$ is zero.

Proof. The proof is by induction on $i \geqslant 0$. The case $i=0$ is trivial, so we assume the statement for $i-1$ and prove it for $i$. We choose $v$ such that for every $0 \leqslant t \leqslant i$ and every $p^{v}$-special homomorphism $f:\left\{P_{m}\right\} \rightarrow\left\{M_{m}\right\}$, the induced map of pro-abelian groups

$$
f_{*}: F_{t}\left(\left\{P_{m}\right\}\right) \rightarrow F_{t}\left(\left\{M_{m}\right\}\right)
$$


is zero. We let $\left\{P_{m}\right\}$ be a pseudo-free $\left\{I^{m}\right\}$-module and choose a $p^{v}$-special augmented complex of $\left\{I^{m}\right\}$-modules $\epsilon:\left\{C_{m, *}\right\} \rightarrow\left\{P_{m}\right\}$. It follows from Prop. 1.6] that this augmented complex is exact as an augmented complex of pro-abelian groups. We let $\epsilon:\left\{P_{m}[-]\right\} \rightarrow\left\{P_{m}\right\}$ be the augmented simplicial $\left\{I^{m}\right\}$-module associated with the augmented complex of $\left\{I^{m}\right\}$-modules $\epsilon:\left\{C_{m, *}\right\} \rightarrow\left\{P_{m}\right\}$ by the Dold-Kan correspondence 29. By assumption, we then have the following spectral sequence of pro-abelian groups:

$$
E_{s, t}^{1}=F_{t}\left(\left\{P_{m}[s]\right\}\right) \Rightarrow F_{s+t}\left(\left\{P_{m}\right\}\right) .
$$

The inductive hypothesis shows that the pro-abelian groups $E_{s, t}^{1}$ are zero, for all $s$ and all $0 \leqslant t<i$. Hence, the edge-homomorphism

$$
F_{i}\left(\left\{P_{m}[0]\right\}\right) \rightarrow F_{i}\left(\left\{P_{m}\right\}\right)
$$

is an epimorphism of pro-abelian groups. But the edge-homomorphism is equal to the map induced by the special homomorphism $\epsilon:\left\{P_{m}[0]\right\} \rightarrow\left\{P_{m}\right\}$ and therefore is zero by assumption. This completes the proof.

If a group $G$ acts from the left on a group $H$, the semi-direct product group $G \ltimes H$ is defined to be the set $G \times H$ with multiplication

$$
(g, h) \cdot\left(g^{\prime}, h^{\prime}\right)=\left(g g^{\prime},\left(g^{\prime-1} h\right) h^{\prime}\right) .
$$

The projection $\pi: G \ltimes H \rightarrow G$ and the section $\sigma: G \rightarrow G \ltimes H$ defined by $\sigma(g)=$ $(g, 1)$ are both group-homomorphisms, and hence, the homology of the semi-direct product group decomposes canonically as a direct sum

$$
H_{q}(G \ltimes H, \mathbb{Z} / p) \stackrel{\sim}{\leftarrow} H_{q}(G, \mathbb{Z} / p) \oplus H_{q}(G \ltimes H, G, \mathbb{Z} / p) .
$$

We write $\mathbb{Z}[X]$ for the free abelian group generated by the set $X$.

Proposition 1.8. Let $\left\{G_{m}\right\}$ be a pro-group, let $\epsilon:\left\{H_{m}[-]\right\} \rightarrow\left\{H_{m}\right\}$ be a strict augmented simplicial pro-group with a strict left $\left\{G_{m}\right\}$-action, and suppose that the associated complex of pro-abelian groups

$$
\cdots \rightarrow\left\{\mathbb{Z}\left[H_{m}[2]\right]\right\} \rightarrow\left\{\mathbb{Z}\left[H_{m}[1]\right]\right\} \rightarrow\left\{\mathbb{Z}\left[H_{m}[0]\right]\right\} \stackrel{\epsilon}{\rightarrow}\left\{\mathbb{Z}\left[H_{m}\right]\right\} \rightarrow 0
$$

is exact. Then there is a natural spectral sequence of pro-abelian groups

$$
E_{s, t}^{1}=\left\{H_{t}\left(G_{m} \ltimes H_{m}[s], G_{m}, \mathbb{Z} / p\right)\right\} \Rightarrow\left\{H_{s+t}\left(G_{m} \ltimes H_{m}, G_{m}, \mathbb{Z} / p\right)\right\},
$$

and the edge-homomorphism

$$
\left\{H_{t}\left(G_{m} \ltimes H_{m}[0], G_{m}, \mathbb{Z} / p\right)\right\} \rightarrow\left\{H_{t}\left(G_{m} \ltimes H_{m}, G_{m}, \mathbb{Z} / p\right)\right\}
$$

is equal to the map induced from $\epsilon$.

Proof. Suppose first that $G$ is a group and that $M_{*}$ is a chain complex of left $G$-modules. The hyper-homology of $G$ with coefficients in $M_{*}$ is defined to be the homology $H_{*}\left(G ; M_{*}\right)$ of the total complex $C_{*}\left(G ; M_{*}\right)$ of the double-complex defined by the bar-construction $B_{*}\left(\mathbb{Z}[G], \mathbb{Z}[G], M_{*}\right)$. The filtration in the bar-direction gives rise to a functorial spectral sequence

$$
E_{s, t}^{2}=H_{s}\left(G ; H_{t}\left(M_{*}\right)\right) \Rightarrow H_{s+t}\left(G ; M_{*}\right),
$$

where $H_{t}\left(M_{*}\right)$ is considered as a complex concentrated in degree zero.

If $K$ is a subgroup of $G$, the complex $C_{*}\left(G, K ; M_{*}\right)$ is defined to be the mapping cone of the map $C_{*}\left(K ; M_{*}\right) \rightarrow C_{*}\left(G ; M_{*}\right)$ induced by the inclusion. We write $\tilde{C}_{*}\left(G ; M_{*}\right)$ instead of $C_{*}\left(G,\{1\} ; M_{*}\right)$. Suppose that $G$ acts from the left on a group 
$H$, and that $M_{*}$ is a complex of left $G \ltimes H$-modules. Then there is a canonical isomorphism of complexes

$$
C_{*}\left(G \ltimes H ; M_{*}\right) \stackrel{\sim}{\leftarrow} C_{*}\left(G ; C_{*}(H ; M)\right)
$$

where the $G$-action on $C_{*}\left(H ; M_{*}\right)$ is induced from the actions by $G$ on $H$ and $M_{*}$. This isomorphism induces an isomorphism of complexes

$$
C_{*}\left(G \ltimes H, G ; M_{*}\right) \stackrel{\sim}{\leftarrow} C_{*}\left(G ; \tilde{C}_{*}\left(H ; M_{*}\right)\right) .
$$

In the following, we consider this isomorphism only for $M_{*}=\mathbb{Z} / p$.

Let $\epsilon: H[-] \rightarrow H$ be an augmented simplicial group with a left action by the group $G$, and let $\tilde{C}_{*}(H[-] ; \mathbb{Z} / p)_{*}$ be the total-complex of the simplicial complex $\tilde{C}_{*}(H[-] ; \mathbb{Z} / p)$. Then the filtration in the simplicial direction gives rise to a functorial spectral sequence of hyper-homology groups

$$
E_{s, t}^{1}=H_{t}\left(G ; \tilde{C}_{*}(H[s] ; \mathbb{Z} / p)\right) \Rightarrow H_{s+t}\left(G ; \tilde{C}_{*}(H[-] ; \mathbb{Z} / p)_{*}\right) .
$$

Suppose now that $\left\{G_{m}\right\}$ and $\epsilon:\left\{H_{m}[-]\right\} \rightarrow\left\{H_{m}\right\}$ are as in the statement. Then we obtain a spectral sequence of strict pro-abelian groups

$$
E_{s, t}^{1}=\left\{H_{t}\left(G_{m} ; \tilde{C}_{*}\left(H_{m}[s] ; \mathbb{Z} / p\right)\right)\right\} \Rightarrow\left\{H_{s+t}\left(G_{m} ; \tilde{C}_{*}\left(H_{m}[-] ; \mathbb{Z} / p\right)_{*}\right)\right\}
$$

which we claim has the desired form. Indeed, the isomorphism of complexes that we established above identifies

$$
\left\{H_{t}\left(G_{m} ; \tilde{C}_{*}\left(H_{m}[s] ; \mathbb{Z} / p\right)\right)\right\} \stackrel{\sim}{\rightarrow}\left\{H_{t}\left(G_{m} \ltimes H_{m}[s], G_{m} ; \mathbb{Z} / p\right)\right\}
$$

and

$$
\left\{H_{s+t}\left(G_{m} ; \tilde{C}_{*}\left(H_{m} ; \mathbb{Z} / p\right)\right)\right\} \stackrel{\sim}{\rightarrow}\left\{H_{s+t}\left(G_{m} \ltimes H_{m}, G_{m} ; \mathbb{Z} / p\right)\right\} .
$$

Hence, it remains only to show that the map induced by the augmentation

$$
\epsilon_{*}:\left\{H_{s+t}\left(G_{m} ; \tilde{C}_{*}\left(H_{m}[-] ; \mathbb{Z} / p\right)_{*}\right)\right\} \rightarrow\left\{H_{s+t}\left(G_{m} ; \tilde{C}_{*}\left(H_{m} ; \mathbb{Z} / p\right)\right)\right\}
$$

is an isomorphism of pro-abelian groups. But the hypothesis of the statement implies that for all $t$, the map induced by the augmentation

$$
\epsilon_{*}:\left\{H_{t}\left(\tilde{C}_{*}\left(H_{m}[-] ; \mathbb{Z} / p\right)_{*}\right)\right\} \rightarrow\left\{\tilde{H}_{t}\left(H_{m} ; \mathbb{Z} / p\right)\right\}
$$

is an isomorphism of pro-abelian groups, and the desired isomorphism then follows from the spectral sequence at the beginning of the proof.

Let again $A$ be a unital associative ring, let $I \subset A$ be a two-sided ideal, and let $\left\{M_{m}\right\}$ be a left $\left\{I^{m}\right\}$-module. Then the group of column vectors $M_{\infty, 1}\left(M_{m}\right)$ is naturally a left $G L\left(I^{m}\right)$-module, and we consider the canonical inclusion of $G L\left(I^{m}\right)$ in the semi-direct product $G L\left(I^{m}\right) \ltimes M_{\infty, 1}\left(M_{m}\right)$. The relative homology groups form a sequence of non-additive functors

$$
F_{i}\left(\left\{M_{m}\right\}\right)=\left\{H_{i}\left(G L\left(I^{m}\right) \ltimes M_{\infty, 1}\left(M_{m}\right), G L\left(I^{m}\right), \mathbb{Z} / p\right)\right\}
$$

from the category of left $\left\{I^{m}\right\}$-modules to the category of pro-abelian groups. This sequence of functors trivially satisfies the condition (i) of Prop. 1.7 and it follows from Prop. 1.8 that it also satisfies the condition (ii). Finally, it follows from 25 , Cor. 6.6] that it satisfies the condition (iii). We now prove the following result which we used in the proof of Prop. 1.3 . 
Proposition 1.9. Let $A$ be a unital associative ring and let $I \subset A$ be a twosided ideal. Let $p$ be a prime and assume that for every positive integer $q$, the following pro-abelian group is zero:

$$
\left\{\operatorname{Tor}_{q}^{\mathbb{Z} \ltimes I^{m}}(\mathbb{Z}, \mathbb{Z} / p)\right\}_{m \geqslant 1} .
$$

Then for all integers $q$, the pro-abelian group

$$
\left\{H_{q}\left(G L\left(I^{m}\right) \ltimes M_{\infty, 1}\left(I^{m}\right), G L\left(I^{m}\right), \mathbb{Z} / p\right)\right\}
$$

is zero.

Proof. Indeed, this follows from Prop. 1.7 since the $\left\{I^{m}\right\}$-module $\left\{I^{m}\right\}$ is pseudo-free.

\section{Topological cyclic homology}

This section is devoted to the proof of the following analog of Thm 1.1 for topological cyclic homology.

THEOREM 2.1. Let $f: A \rightarrow B$ be a map of unital associative rings, and let $I \subset A$ be a two-sided ideal such that $f: I \rightarrow f(I)$ is an isomorphism onto a twosided ideal of $B$. Let $p$ be a prime and suppose that for all positive integers $q$, the pro-abelian group

$$
\left\{\operatorname{Tor}_{q}^{\mathbb{Z} \ltimes I^{m}}(\mathbb{Z}, \mathbb{Z} / p)\right\}_{m \geqslant 1}
$$

is zero. Then for all integers $q$, and all positive integers $n$ and $v$, the induced map of pro-abelian groups

$$
\left\{\mathrm{TC}_{q}^{n}\left(A, I^{m} ; p, \mathbb{Z} / p^{v}\right)\right\}_{m \geqslant 1} \rightarrow\left\{\mathrm{TC}_{q}^{n}\left(B, f(I)^{m} ; p, \mathbb{Z} / p^{v}\right)\right\}_{m \geqslant 1}
$$

is an isomorphism.

The proof is similar, in outline, to the original proof by Wodzicki 30 of excision in cyclic homology for $H$-unital rings, but the technical details are somewhat different. We first show, as in the original case, that the statement for topological cyclic homology follows from the analogous statement for topological Hochschild homology. We briefly recall topological Hochschild homology and topological cyclic homology. The reader is referred to [18 Sect. 1] and [15] for a more detailed account.

The topological Hochschild spectrum $T(A)$ of a unital associated ring $A$ is a cyclotomic spectrum in the sense of [16. Def. 2.2]. In particular, $T(A)$ is an object of the $\mathbb{T}$-stable homotopy category, where $\mathbb{T}$ is the circle group, and is equipped with additional cyclotomic structure maps which on the level of the equivariant homotopy groups

$$
\operatorname{TR}_{q}^{n}(A ; p)=\left[S^{q} \wedge\left(\mathbb{T} / C_{p^{n-1}}\right)_{+}, T(A)\right]_{\mathbb{T}}
$$

give rise to the so-called restriction maps

$$
R: \operatorname{TR}_{q}^{n}(A ; p) \rightarrow \mathrm{TR}_{q}^{n-1}(A ; p) .
$$

We consider the family of groups $\left\{\mathrm{TR}_{q}^{n}(A ; p)\right\}_{n \geqslant 1}$ as a pro-abelian group with the restriction maps as the structure maps. As a consequence of the cyclotomic spectrum structure, there are natural long-exact sequences

$$
\cdots \rightarrow \mathbb{H}_{q}\left(C_{p^{n-1}}, T(A)\right) \rightarrow \mathrm{TR}_{q}^{n}(A ; p) \stackrel{R}{\rightarrow} \operatorname{TR}_{q}^{n-1}(A ; p) \rightarrow \cdots .
$$


The group $\operatorname{TR}_{q}^{1}(A ; p)$ is the $q$ th topological Hochschild group $\mathrm{TH}_{q}(A)$, and there are and natural spectral sequences

$$
E_{s, t}^{2}=H_{s}\left(C_{p^{n-1}}, \mathrm{TH}_{t}(A)\right) \Rightarrow \mathbb{H}_{s+t}\left(C_{p^{n-1}}, T(A)\right) .
$$

The canonical projection $\mathbb{T} / C_{p^{n-2}} \rightarrow \mathbb{T} / C_{p^{n-1}}$ induces the Frobenius map

$$
F: \operatorname{TR}_{q}^{n}(A ; p) \rightarrow \operatorname{TR}_{q}^{n-1}(A ; p),
$$

and the topological cyclic homology groups $\operatorname{TC}_{q}^{n}(A ; p)$ are defined so that there is a natural long-exact sequence of abelian groups

$$
\cdots \rightarrow \mathrm{TC}_{q}^{n}(A ; p) \rightarrow \mathrm{TR}_{q}^{n}(A ; p) \stackrel{R-F}{\longrightarrow} \mathrm{TR}_{q}^{n-1}(A ; p) \stackrel{\partial}{\rightarrow} \mathrm{TC}_{q-1}^{n}(A ; p) \rightarrow \cdots .
$$

Let $I \subset A$ be a two-sided ideal. Then one has the following natural distinguished triangle in the $\mathbb{T}$-stable category:

$$
T(A, I) \rightarrow T(A) \rightarrow T(A / I) \stackrel{\partial}{\rightarrow} \Sigma T(A, I) .
$$

Moreover, Bökstedt and Madsen [3 Prop. 10.2] have given an explicit construction of this distinguished triangle with the property that it is also a sequence of cyclotomic spectra. It follows that there are induced natural long-exact sequences

$$
\cdots \rightarrow \mathrm{TC}_{q}^{n}(A, I ; p) \rightarrow \mathrm{TC}_{q}^{n}(A ; p) \rightarrow \mathrm{TC}_{q}^{n}(A / I ; p) \stackrel{\partial}{\rightarrow} \mathrm{TC}_{q-1}^{n}(A, I ; p) \rightarrow \cdots
$$

and that there are relative versions of the long-exact sequences and the spectral sequence considered above. Hence, to prove Thm. 2.1] above, it suffices to prove the following analogous result for topological Hochschild homology. We remark that this statement concerns only the underlying non-equivariant spectrum $\mathrm{TH}(A, I)$ of the $\mathbb{T}$-equivariant spectrum $T(A, I)$.

TheOREm 2.2. Let $f: A \rightarrow B$ be a map of unital associative rings, and let $I \subset A$ be a two-sided ideal such that $f: I \rightarrow f(I)$ is an isomorphism onto a twosided ideal of $B$. Let $p$ be a prime, and suppose that for all positive integers $q$, the pro-abelian group

$$
\left\{\operatorname{Tor}_{q}^{\mathbb{Z} \ltimes I^{m}}(\mathbb{Z}, \mathbb{Z} / p)\right\}_{m \geqslant 1}
$$

is zero. Then for all integers $q$, the induced map

$$
\left\{\mathrm{TH}_{q}\left(A, I^{m}, \mathbb{Z} / p\right)\right\}_{m \geqslant 1} \rightarrow\left\{\mathrm{TH}_{q}\left(B, f(I)^{m}, \mathbb{Z} / p\right)\right\}_{m \geqslant 1}
$$

is an isomorphism of pro-abelian groups.

The proof of Thm. 2.2 is rather lengthy and a brief outline is in order. The definition of topological Hochschild homology extends to non-unital rings and there is a canonical map $\mathrm{TH}(I) \rightarrow \mathrm{TH}(A, I)$. We show that the mapping cone of this map is $B(I)$-cellular [19 Def. 5.1.4], where $B(I)$ is the bar-construction of the Eilenberg-MacLane spectrum of $I$. The precise statement is given in Lemma 2.7 below. Finally, we relate the $\mathbb{Z} / p$-homology groups of the spectrum $B(I)$ to the groups $\operatorname{Tor}_{q}^{\mathbb{Z} \ltimes I}(\mathbb{Z}, \mathbb{Z} / p)$ by means of a spectral sequence. We then conclude that for all integers $q$, the canonical map

$$
\left\{\mathrm{TH}_{q}\left(I^{m}, \mathbb{Z} / p\right)\right\}_{m \geqslant 1} \rightarrow\left\{\mathrm{TH}_{q}\left(A, I^{m}, \mathbb{Z} / p\right)\right\}_{m \geqslant 1}
$$

is an isomorphism of pro-abelian groups. Hence, the right-hand side is independent of the ring $A$ as stated in Thm. 2.2.

We recall from [20 that the category of symmetric spectra is a closed model category and that the associated homotopy category is the stable homotopy category. Moreover, the smash product of symmetric spectra defines a closed symmetric 
monoidal structure which induces a closed symmetric monoidal structure on the stable homotopy category.

We will consider various symmetric spectra that are defined as the realization of semi-simplicial symmetric spectra. To briefly recall this, let $\boldsymbol{\Delta}_{\text {mon }}$ be the category whose objects are the finite ordered sets

$$
[k]=\{1,2, \ldots, k\}, \quad k \geqslant 0,
$$

and whose morphisms are all injective order-preserving maps. The morphisms are generated by the coface maps $d^{r}:[k-1] \rightarrow[k], 0 \leqslant r \leqslant k$, defined by $d^{r}(i)=i$, for $0 \leqslant i<r$, and $d^{r}(i)=i+1$, for $r \leqslant i<k$. Then a semi-simplicial symmetric spectrum is a functor $X[-]$ from $\boldsymbol{\Delta}_{\text {mon }}^{\mathrm{op}}$ to the category of symmetric spectra, and the realization is the symmetric spectrum given by the coequalizer

$$
\bigvee X\left[k^{\prime}\right] \wedge\left(\Delta^{k}\right)_{+} \longrightarrow \bigvee X[k] \wedge\left(\Delta^{k}\right)_{+} \longrightarrow\|[k] \mapsto X[k]\|,
$$

where the wedge-sums in the middle and on the left-hand side range over all objects and morphisms, respectively, of $\boldsymbol{\Delta}_{\text {mon }}$. The two maps are given, on the summand indexed by the morphism $\theta:[k] \rightarrow\left[k^{\prime}\right]$, by the maps $X\left[k^{\prime}\right] \wedge \theta_{*}$ and $\theta^{*} \wedge\left(\Delta^{k}\right)_{+}$, respectively. The skeleton filtration of the realization gives rise to a natural spectral sequence

$$
E_{s, t}^{1}=\pi_{t}(X[s]) \Rightarrow \pi_{s+t}(\|[k] \mapsto X[k]\|)
$$

where the $d^{1}$-differential is given by the alternating sum of the maps induced by the face maps $d_{r}: X[s] \rightarrow X[s-1], 0 \leqslant r \leqslant s$.

Let $I$ be a non-unital associative ring. We abuse notation and write $I$ also for the associated Eilenberg-MacLane spectrum [20 Ex. 1.2.5]. The ring structure gives rise to a map of symmetric spectra

$$
\mu: I \wedge I \rightarrow I
$$

that makes $I$ a non-unital associative symmetric ring spectrum. We define the topological Hochschild spectrum of $I$ to be the symmetric spectrum

$$
\mathrm{TH}(I)=\|[k] \mapsto \mathrm{TH}(I)[k]\|
$$

that is the realization of the semi-simplicial symmetric spectrum defined as follows. The symmetric spectrum in degree $k$ is the $(k+1)$-fold smash product

$$
\mathrm{TH}(I)[k]=I^{\wedge(k+1)}
$$

and the face maps $d_{r}: \operatorname{TH}(I)[k] \rightarrow \operatorname{TH}(I)[k-1], 0 \leqslant r \leqslant k$, are defined by

$$
d_{r}= \begin{cases}I^{\wedge r} \wedge \mu \wedge I^{\wedge(k-1-r)} & 0 \leqslant r<k, \\ \left(\mu \wedge I^{\wedge(k-1)}\right) \circ \tau & r=k .\end{cases}
$$

Here $\tau$ is the canonical isomorphism that switches the first $k$ smash factors and the last smash factor. We recall from 24, Thm. 4.2.8] that if $A$ is a unital associative ring, the symmetric spectrum $\mathrm{TH}(A)$ defined here is canonically weakly equivalent to the topological Hochschild spectrum defined by Bökstedt. We note, however, that the symmetric spectrum $\mathrm{TH}(A)$ defined here is not equipped with the additional cyclotomic structure that is needed for the definition of topological cyclic homology. But this extra structure is not relevant for the proof of Thm. 2.2

Let $A$ be a unital associative ring, and let $I \subset A$ be a two-sided ideal. We continue to write $A$ and $I$ for the associated Eilenberg-MacLane spectra. Then $A$ 
is a unital associative symmetric ring spectrum, $I$ is a unital and symmetric $A$ - $A$ bimodule spectrum, and the map $\rho: I \rightarrow A$ induced by the inclusion of $I$ in $A$ is a map of $A$ - $A$-bimodule spectra. We follow Bökstedt-Madsen [3, Appendix] and define a symmetric spectrum

$$
\mathrm{TH}(A)^{\prime}=\left\|[k] \mapsto \mathrm{TH}(A)^{\prime}[k]\right\|
$$

as the realization of a semi-simplicial symmetric spectrum that we now define. Let $\mathcal{P}[k]$ be the category with objects all tuples $\epsilon=\left(\epsilon_{0}, \ldots, \epsilon_{k}\right)$ of integers $\epsilon_{i} \in\{0,1\}$ and with a single morphism from $\epsilon$ to $\epsilon^{\prime}$ if $\epsilon_{i} \geqslant \epsilon_{i}^{\prime}$, for all $0 \leqslant i \leqslant k$. So $\mathcal{P}[k]$ is an index category for $(k+1)$-dimensional cubical diagrams. We let $E[k]$ be the functor from $\mathcal{P}[k]$ to the category of symmetric spectra that is given on objects and morphisms, respectively, by

$$
\begin{aligned}
& E[k]\left(\epsilon_{0}, \ldots, \epsilon_{r}, \ldots, \epsilon_{k}\right)=E^{\epsilon_{0}} \wedge \cdots \wedge E^{\epsilon_{r}} \wedge \cdots \wedge E^{\epsilon_{k}} \\
& E[k]\left(\epsilon_{0}, \ldots, \iota_{r}, \ldots, \epsilon_{k}\right)=E^{\epsilon_{0}} \wedge \cdots \wedge \rho \wedge \cdots \wedge E^{\epsilon_{k}},
\end{aligned}
$$

where $E^{0}=A$ and $E^{1}=I$, and where $\left(\epsilon_{0}, \ldots, \iota_{r}, \ldots, \epsilon_{k}\right)$ denotes the unique morphism from $\left(\epsilon_{0}, \ldots, 1, \ldots, \epsilon_{k}\right)$ to $\left(\epsilon_{0}, \ldots, 0, \ldots, \epsilon_{k}\right)$. Then

$$
\operatorname{TH}(A)^{\prime}[k]=\underset{\mathcal{P}[k]}{\operatorname{hocolim}} E[k] ;
$$

see [4, Chap. XII] for the definition of homotopy colimits. To define the face maps, we first note that the ring and bimodule spectrum maps define maps of symmetric spectra $\mu_{\epsilon, \epsilon^{\prime}}: E^{\epsilon} \wedge E^{\epsilon^{\prime}} \rightarrow E^{\epsilon \sqcup \epsilon^{\prime}}$ where $\epsilon \sqcup \epsilon^{\prime}=\max \left\{\epsilon, \epsilon^{\prime}\right\}$. Then the face maps

$$
d_{r}: \operatorname{TH}(A)^{\prime}[k] \rightarrow \operatorname{TH}(A)^{\prime}[k-1], \quad 0 \leqslant r \leqslant k,
$$

are defined to be the composites

$$
\underset{\mathcal{P}[k]}{\operatorname{hocolim}} E[k] \stackrel{\delta_{r}}{\rightarrow} \underset{\mathcal{P}[k]}{\operatorname{hocolim}} E[k-1] \circ \mathfrak{d}_{r} \rightarrow \underset{\mathcal{P}[k-1]}{\operatorname{hocolim}} E[k-1],
$$

where $\mathfrak{d}_{r}: \mathcal{P}[k] \rightarrow \mathcal{P}[k-1]$ is the functor

$$
\mathfrak{d}_{r}\left(\epsilon_{0}, \ldots, \epsilon_{k}\right)= \begin{cases}\left(\epsilon_{0}, \ldots, \epsilon_{r} \sqcup \epsilon_{r+1}, \epsilon_{k}\right) & 0 \leqslant r<k, \\ \left(\epsilon_{k} \sqcup \epsilon_{0}, \epsilon_{1}, \ldots, \epsilon_{k-1}\right) & r=k,\end{cases}
$$

and where $\delta_{r}: E[k] \rightarrow E[k-1] \circ \mathfrak{d}_{r}$ is the natural transformation

$$
\delta_{r}= \begin{cases}E^{\epsilon_{0}} \wedge \cdots \wedge E^{\epsilon_{r-1}} \wedge \mu_{\epsilon_{r}, \epsilon_{r+1}} \wedge E^{\epsilon_{r+2}} \wedge \cdots \wedge E^{\epsilon_{k}} & 0 \leqslant r<k, \\ \left(\mu_{\epsilon_{k}, \epsilon_{0}} \wedge E^{\epsilon_{1}} \wedge \cdots \wedge E^{\epsilon_{k-1}}\right) \circ \tau & r=k .\end{cases}
$$

The object $(0, \ldots, 0)$ in $\mathcal{P}[k]$ is a final object, and hence, the canonical map

$$
\mathrm{TH}(A)[k]=E[k](0, \ldots, 0) \rightarrow \underset{\mathcal{P}[k]}{\operatorname{hocolim}} E[k]=\mathrm{TH}(A)^{\prime}[k]
$$

is a weak equivalence 4, XII.3.1]. The canonical maps form a map of semi-simplicial symmetric spectra and the spectral sequence (2.4) shows that the induced map of realizations

$$
\mathrm{TH}(A) \rightarrow \mathrm{TH}(A)^{\prime}
$$

is a weak equivalence of symmetric spectra.

Let $\mathcal{P}^{\prime}[k] \subset \mathcal{P}[k]$ be the full subcategory whose objects are the tuples $\epsilon$ such that not all of $\epsilon_{0}, \ldots, \epsilon_{k}$ are equal to zero. The symmetric spectrum

$$
\operatorname{TH}(A, I)[k]=\underset{\mathcal{P}^{\prime}[k]}{\operatorname{hocolim}} E[k]
$$


is a sub-symmetric spectrum of $\operatorname{TH}(A)^{\prime}[k]$, and there is a canonical weak equivalence from the mapping cone of the inclusion onto the symmetric spectrum $\mathrm{TH}(A / I)[k]$. Indeed, this follows from the proof of Lemma 2.5below. The face maps $d_{r}: \operatorname{TH}(A)^{\prime}[k] \rightarrow$ $\operatorname{TH}(A)^{\prime}[k-1], 0 \leqslant r \leqslant k$, restrict to face maps $d_{r}: \operatorname{TH}(A, I)[k] \rightarrow \operatorname{TH}(A, I)[k-1]$, $0 \leqslant r \leqslant k$, and we define

$$
\operatorname{TH}(A, I)=\|[k] \mapsto \operatorname{TH}(A, I)[k]\|
$$

to be the realization. Then from the canonical weak equivalences of $\operatorname{TH}(A)$ and $\mathrm{TH}(A)^{\prime}$ and of $\mathrm{TH}(A / I)$ and the mapping cone of the canonical inclusion of $\mathrm{TH}(A, I)$ in $\mathrm{TH}(A)^{\prime}$ we obtain the following distinguished triangle in the stable homotopy category:

$$
\mathrm{TH}(A, I) \rightarrow \mathrm{TH}(A) \rightarrow \mathrm{TH}(A / I) \stackrel{\partial}{\rightarrow} \Sigma \mathrm{TH}(A, I) .
$$

This distinguished triangle is canonically isomorphic to the distinguished triangle underlying the distinguished triangle of $\mathbb{T}$-spectra of [3] Prop. 10.2].

The category $\mathcal{P}^{\prime}[k]$ admits a finite filtration

$$
\emptyset \subset \operatorname{Fil}_{0} \mathcal{P}^{\prime}[k] \subset \operatorname{Fil}_{1} \mathcal{P}^{\prime}[k] \subset \cdots \subset \operatorname{Fil}_{k-1} \mathcal{P}^{\prime}[k] \subset \operatorname{Fil}_{k} \mathcal{P}^{\prime}[k]=\mathcal{P}^{\prime}[k]
$$

with $\mathrm{Fil}_{s} \mathcal{P}^{\prime}[k]$ the full sub-category with objects the tuples $\left(\epsilon_{0}, \ldots, \epsilon_{k}\right)$ such that at most $s$ of $\epsilon_{0}, \ldots, \epsilon_{k}$ are equal to 0 . We obtain an induced filtration

$$
* \subset \operatorname{Fil}_{0} \mathrm{TH}(A, I)[k] \subset \operatorname{Fil}_{1} \mathrm{TH}(A, I)[k] \subset \cdots \subset \mathrm{TH}(A, I)[k]
$$

where $\operatorname{Fil}_{s} \mathrm{TH}(A, I)[k]$ is the sub-symmetric spectrum defined to be the homotopy colimit of the restriction of the functor $E[k]$ to $\operatorname{Fil}_{s} \mathcal{P}^{\prime}[k]$. The functor $\mathfrak{d}_{r}: \mathcal{P}^{\prime}[k] \rightarrow$ $\mathcal{P}^{\prime}[k-1]$ takes $\mathrm{Fil}_{s} \mathcal{P}^{\prime}[k]$ to $\mathrm{Fil}_{s} \mathcal{P}^{\prime}[k-1]$, for $0 \leqslant s<k$, and therefore, the face map $d_{r}: \operatorname{TH}(A, I)[k] \rightarrow \operatorname{TH}(A, I)[k-1]$ takes $\operatorname{Fil}_{s} \operatorname{TH}(A, I)[k]$ to $\operatorname{Fil}_{s} \operatorname{TH}(A, I)[k-1]$, for $0 \leqslant s<k$. Hence, we can form the realization

$$
\operatorname{Fil}_{s} \operatorname{TH}(A, I)=\left\|[k] \mapsto \operatorname{Fil}_{s} \operatorname{TH}(A, I)[k]\right\|
$$

and obtain the following filtration of $\operatorname{TH}(A, I)$ by sub-symmetric spectra:

$$
* \subset \operatorname{Fil}_{0} \operatorname{TH}(A, I) \subset \operatorname{Fil}_{1} \operatorname{TH}(A, I) \subset \operatorname{Fil}_{2} \operatorname{TH}(A, I) \subset \cdots \subset \mathrm{TH}(A, I) .
$$

We define the filtration quotient $\operatorname{gr}_{s} \mathrm{TH}(A, I)[k]$ to be the mapping cone of the canonical inclusion of $\operatorname{Fil}_{s-1} \mathrm{TH}(A, I)[k]$ in $\operatorname{Fil}_{s} \mathrm{TH}(A, I)[k]$ and let

$$
\operatorname{gr}_{s} \operatorname{TH}(A, I)=\left\|[k] \mapsto \operatorname{gr}_{s} \operatorname{TH}(A, I)[k]\right\| .
$$

Since forming mapping cone and realization commute, up to canonical isomorphism, $\operatorname{gr}_{s} \operatorname{TH}(A, I)$ is canonically isomorphic to the mapping cone of the inclusion of $\mathrm{Fil}_{s-1} \mathrm{TH}(A, I)$ in $\mathrm{Fil}_{s} \mathrm{TH}(A, I)$. We evaluate the homotopy type of $\operatorname{gr}_{s} \mathrm{TH}(A, I)$ and begin by evaluating that of $\operatorname{gr}_{s} \mathrm{TH}(A, I)[k]$.

Let $E^{1} \rightarrow E^{0}$ and $E^{0} \rightarrow E^{-1}$ be the maps of unital symmetric $A$ - $A$-bimodule spectra associated with the canonical inclusion of $I$ in $A$ and the canonical projection of $A$ onto $A / I$. We call these maps the canonical maps. We say that a tuple $\epsilon=\left(\epsilon_{0}, \ldots, \epsilon_{k}\right)$ of integers $\epsilon_{i} \in\{1,0,-1\}$ is a weight of rank $k$ and write $|\epsilon|_{v}$ for the number of $0 \leqslant i \leqslant k$ with $\epsilon_{i}=v$. We have the following analog of the hyper-cube lemma [30, Lemma A.1]:

Lemma 2.5. For every $0 \leqslant s \leqslant k$, there is a canonical weak equivalence

$$
\bar{\Phi}_{s}: \operatorname{gr}_{s} \operatorname{TH}(A, I)[k] \stackrel{\sim}{\rightarrow} \bigvee E^{\epsilon_{0}} \wedge \cdots \wedge E^{\epsilon_{k}}
$$


where, on the right-hand side, the wedge sum ranges over all weights $\epsilon$ of rank $k$ with $|\epsilon|_{0}=0$ and $|\epsilon|_{-1}=s$.

Proof. There is a partial ordering on the set of weights of rank $k$ where $\epsilon \preccurlyeq \epsilon^{\prime}$ if $\epsilon_{i} \geqslant \epsilon_{i}^{\prime}$, for all $0 \leqslant i \leqslant k$. We first define a map of symmetric spectra

$$
\Phi_{s}: \operatorname{Fil}_{s} \operatorname{TH}(A, I)[k]=\underset{\operatorname{Fil}_{s} \mathcal{P}^{\prime}[k]}{\operatorname{hocolim}} E^{\epsilon_{0}} \wedge \cdots \wedge E^{\epsilon_{k}} \rightarrow \prod E^{\epsilon_{0}} \wedge \cdots \wedge E^{\epsilon_{k}}
$$

where the product on the right-hand side is indexed by the set of weights of rank $k$ with $|\epsilon|_{0}=0$ and $|\epsilon|_{-1}=s$. We recall from [4, XII.2.2] that to give the map $\Phi_{s}$ is equivalent to giving, for each weight $\epsilon$ of rank $k$ with $|\epsilon|_{0}=0$ and $|\epsilon|_{-1}=s$, a compatible family of maps

$$
\Phi_{s, \epsilon, \epsilon^{\prime}}:\left(E^{\epsilon_{0}^{\prime}} \wedge \cdots \wedge E^{\epsilon_{k}^{\prime}}\right) \wedge\left|\epsilon^{\prime} / \mathrm{Fil}_{s} \mathcal{P}^{\prime}[k]\right|_{+} \rightarrow E^{\epsilon_{0}} \wedge \cdots \wedge E^{\epsilon_{k}},
$$

where $\epsilon^{\prime}$ ranges over all objects of $\mathrm{Fil}_{s} \mathcal{P}^{\prime}[k]$, and where the second smash factor on the left-hand side is the geometric realization of the nerve of the under-category $\epsilon^{\prime} / \mathrm{Fil}_{s} \mathcal{P}^{\prime}[k]$. Combatibility means that if $\epsilon^{\prime} \preccurlyeq \epsilon^{\prime \prime}$, then the following diagram commutes:

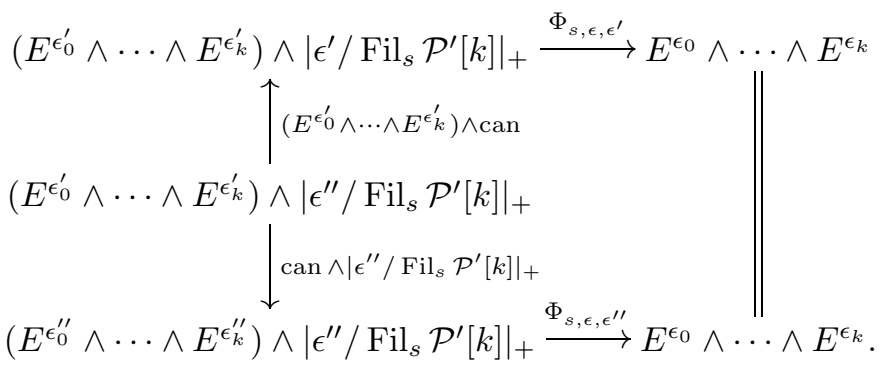

Now, there is a unique weight $\omega$ of rank $k$ with $\omega \preccurlyeq \epsilon$ and $|\omega|_{0}=s$. The category $\omega / \mathrm{Fil}_{s} \mathcal{P}^{\prime}[k]$ has one object and one morphism, and we define

$$
\Phi_{s, \epsilon, \omega}: E^{\omega_{0}} \wedge \cdots \wedge E^{\omega_{k}} \rightarrow E^{\epsilon_{0}} \wedge \cdots \wedge E^{\epsilon_{k}}
$$

to be the canonical map. If $\epsilon^{\prime} \neq \omega$, we define $\Phi_{s, \epsilon, \epsilon^{\prime}}$ to be the constant map. To see that these maps are compatible, suppose that $\epsilon^{\prime} \preccurlyeq \omega$. Then either $\epsilon^{\prime}=\omega$ or $\left|\epsilon^{\prime}\right|_{0}<s$. The former case is trivial, and in the latter case, the composition of the following canonical maps

$$
E^{\epsilon_{0}^{\prime}} \wedge \cdots \wedge E^{\epsilon_{k}^{\prime}} \rightarrow E^{\omega_{0}} \wedge \cdots \wedge E^{\omega_{k}} \rightarrow E^{\epsilon_{0}} \wedge \cdots \wedge E^{\epsilon_{k}}
$$

is equal to the constant map. Indeed, on at least one smash factor, the map is induced by the composition $I \rightarrow A \rightarrow A / I$, which is zero. Hence, we obtain the desired map $\Phi_{s}$. It is clear from the definition that the image of $\Phi_{s}$ is contained in the corresponding wedge sum, and that the restriction of $\Phi_{s}$ to $\operatorname{Fil}_{s-1} \mathrm{TH}(A, I)[k]$ is equal to the constant map. Therefore, we obtain the map $\bar{\Phi}_{s}$ of the statement.

The proof that $\bar{\Phi}_{s}$ is a weak equivalence is by induction on $k \geqslant 0$. The case $k=0$ is trivial, and the induction step uses the following observation. Suppose that $\epsilon^{\prime} \preccurlyeq \epsilon \preccurlyeq \epsilon^{\prime \prime}$ are such that for some $0 \leqslant v \leqslant k, \epsilon^{\prime}(v)=1, \epsilon(v)=0$, and $\epsilon^{\prime \prime}(v)=-1$, and such that for all $0 \leqslant i \leqslant k$ with $i \neq v, \epsilon^{\prime}(i)=\epsilon(i)=\epsilon^{\prime \prime}(i)$. Then the composition of the canonical maps

$$
E^{\epsilon_{0}^{\prime}} \wedge \cdots \wedge E^{\epsilon_{k}^{\prime}} \rightarrow E^{\epsilon_{0}} \wedge \cdots \wedge E^{\epsilon_{k}} \rightarrow E^{\epsilon_{0}^{\prime \prime}} \wedge \cdots \wedge E^{\epsilon_{k}^{\prime \prime}}
$$


is the constant map, and the induced map from the mapping cone of the left-hand map to the right-hand term is a weak equivalence.

We define maps of symmetric spectra

$$
d_{r, s}: \bigvee E^{\epsilon_{0}} \wedge \cdots \wedge E^{\epsilon_{k}} \rightarrow \bigvee E^{\epsilon_{0}} \wedge \cdots \wedge E^{\epsilon_{k-1}}, \quad 0 \leqslant r \leqslant k
$$

where the wedge sum on the left and right-hand side range over all weights $\epsilon$ of rank $k$ and $k-1$, respectively, with $|\epsilon|_{0}=0$ and $|\epsilon|_{-1}=s$. Let $\epsilon$ be a weight of rank $k$ with $|\epsilon|_{0}=0$ and $|\epsilon|_{-1}=s$, and let $0 \leqslant r \leqslant k$. We define a weight $\partial_{r}(\epsilon)$ of rank $k-1$ with $\left|\partial_{r}(\epsilon)\right|_{0}=0$ and with $\left|\partial_{r}(\epsilon)\right|_{-1} \leqslant s$ by

$$
\partial_{r}(\epsilon)_{i}= \begin{cases}\epsilon_{i} & \text { for } 0 \leqslant i<r, \\ \max \left\{\epsilon_{r}, \epsilon_{r+1}\right\} & \text { for } i=r, \\ \epsilon_{i+1} & \text { for } r<i \leqslant k-1,\end{cases}
$$

if $0 \leqslant r<k$, and by

$$
\partial_{k}(\epsilon)_{i}= \begin{cases}\max \left\{\epsilon_{k}, \epsilon_{0}\right\} & \text { for } i=0 \\ \epsilon_{i} & \text { for } 0<i \leqslant k-1\end{cases}
$$

if $r=k$. Then we define the component $d_{r, s, \epsilon}$ of the map $d_{r, s}$ to be the constant map, if $\left|\partial_{r}(\epsilon)\right|_{-1}<s$, and to be the composition

$$
E^{\epsilon_{0}} \wedge \cdots \wedge E^{\epsilon_{k}} \stackrel{\delta_{r}}{\longrightarrow} E^{\partial_{r}(\epsilon)_{0}} \wedge \cdots \wedge E^{\partial_{r}(\epsilon)_{k-1}} \rightarrow \bigvee_{\epsilon^{\prime} \in \mathcal{P}(k-1, s)} E^{\epsilon_{0}^{\prime}} \wedge \cdots \wedge E^{\epsilon_{k-1}^{\prime}},
$$

if $\left|\partial_{r}(\epsilon)\right|_{-1}=s$. Here the right-hand map is the canonical inclusion. The next result is an immediate consequence of the construction of the map $\bar{\Phi}_{s}$ :

AdDEndum 2.6. For all $0 \leqslant s \leqslant k$ and $0 \leqslant r \leqslant k$, the diagram

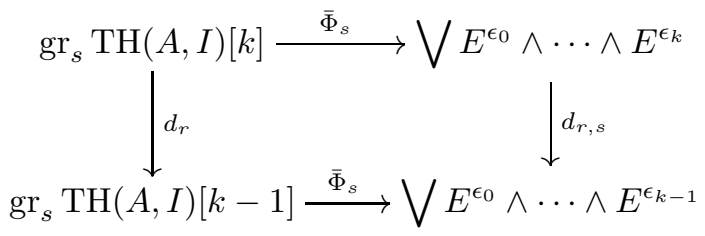

commutes. Here the wedge sums on the right-hand side range over all weights $\epsilon$ of rank $k$ and $k-1$, respectively, with $|\epsilon|_{0}=0$ and $|\epsilon|_{-1}=s$.

We now evaluate the homotopy type of the realization $\operatorname{gr}_{s} \mathrm{TH}(A, I)$. The spectra on the right-hand side in Lemma 2.5 form a semi-simplicial symmetric spectrum, that we denote $\operatorname{gr}_{s} \mathrm{TH}(A, I)^{\dagger}[-]$, with face maps

$$
d_{r, s}: \operatorname{gr}_{s} \mathrm{TH}(A, I)^{\dagger}[k] \rightarrow \operatorname{gr}_{s} \mathrm{TH}(A, I)^{\dagger}[k-1], \quad 0 \leqslant r \leqslant k,
$$

and Addendum 2.6 shows that the weak equivalence of Lemma 2.5 forms a map of semi-simplicial symmetric spectra. The spectral sequence (2.4) shows that the induced map of realizations is a weak equivalence

$$
\bar{\Phi}_{s}: \operatorname{gr}_{s} \operatorname{TH}(A, I) \stackrel{\sim}{\longrightarrow} \operatorname{gr}_{s} \operatorname{TH}(A, I)^{\dagger} .
$$

It is clear that for $s=0$, we have

$$
\operatorname{gr}_{0} \mathrm{TH}(A, I)^{\dagger}=\mathrm{TH}(I) .
$$


We proceed to show that the higher filtration quotients are $B(I)$-cellular, where $B(I)$ is the bar-construction of the non-unital associative symmetric ring spectrum $I$. This is defined to be the realization

$$
B(I)=\|[k] \mapsto B(I)[k]\|
$$

of the semi-simplicial symmetric spectrum with $B(I)[k]=I^{\wedge k}$, if $k>0$, with $B(I)[0]=*$, and with face maps defined by

$$
d_{r}= \begin{cases}I^{\wedge(r-1)} \wedge \mu \wedge I^{\wedge(k-r-1)} & 0<r<k \\ * & r=0 \text { or } r=k .\end{cases}
$$

To this end, for $s>0$, we further decompose

$$
\operatorname{gr}_{s} \operatorname{TH}(A, I)^{\dagger}=\operatorname{gr}_{s} \mathrm{TH}(A, I)_{0}^{\dagger} \vee \operatorname{gr}_{s} \mathrm{TH}(A, I)_{1}^{\dagger}
$$

where the first term on the right-hand side is the realization of the sub-semisimplicial symmetric spectrum that consists of the single wedge summand with weight $\epsilon=(-1, \ldots,-1,1, \ldots, 1)$ and the second is the realization of the sub-semisimplicial symmetric spectrum that consists of the remaining wedge summands. Following [30 Lemma 3.3] we introduce a further filtration of the semi-simplicial symmetric spectrum $\operatorname{gr}_{s} \operatorname{TH}(A, I)_{1}^{\dagger}[-]$. We define

$$
\operatorname{Fil}_{u}^{\prime} \operatorname{gr}_{s} \operatorname{TH}(A, I)_{1}^{\dagger}[k] \subset \operatorname{gr}_{s} \operatorname{TH}(A, I)_{1}^{\dagger}[k]
$$

to consist of the wedge summands indexed by the weights $\epsilon$ for which there exists $k-u \leqslant j \leqslant k$ with $\epsilon_{j}=-1$, and define $\operatorname{gr}_{u}^{\prime} \operatorname{gr}_{s} \operatorname{TH}(A, I)_{1}^{\dagger}[k]$ to be the filtration quotient. After realization, we get an induced filtration of the symmetric spectrum $\operatorname{gr}_{s} \operatorname{TH}(A, I)_{1}^{\dagger}$ with filtration quotients

$$
\operatorname{gr}_{u}^{\prime} \operatorname{gr}_{s} \operatorname{TH}(A, I)_{1}^{\dagger}=\left\|[k] \mapsto \operatorname{gr}_{u}^{\prime} \operatorname{gr}_{s} \operatorname{TH}(A, I)_{1}^{\dagger}[k]\right\| .
$$

We prove that the higher filtration quotients $\operatorname{gr}_{s} \mathrm{TH}(A, I)$ are $B(I)$-cellular in the following precise sense:

LEMmA 2.7. For all positive integers $s$, and all non-negative integers $u$, there are canonical isomorphisms in the stable homotopy category

$$
\begin{gathered}
\Sigma^{s-1}(A / I)^{\wedge s} \wedge B(I) \stackrel{\sim}{\rightarrow} \operatorname{gr}_{s} \operatorname{TH}(A, I)_{0}^{\dagger} \\
\bigvee \Sigma^{s+u-1} B(I)^{\wedge \ell} \wedge(A / I)^{\wedge s} \wedge I^{\wedge u} \stackrel{\sim}{\rightarrow} \operatorname{gr}_{u}^{\prime} \operatorname{gr}_{s} \operatorname{TH}(A, I)_{1}^{\dagger}
\end{gathered}
$$

where the wedge sum ranges over all sequences $\left(n_{0}, \ldots, n_{\ell}\right)$ of non-negative integers of length $\ell \geqslant 1$ such that $n_{0}+\cdots+n_{\ell}=s$, and such that $n_{i}>0$, for $i>0$. The isomorphisms are natural with respect to the pair $(A, I)$.

Proof. To establish the first isomorphism, we note that the symmetric spectrum in simplicial degree $k, \operatorname{gr}_{s} \operatorname{TH}(A, I)_{0}^{\dagger}[k]$, is trivial, for $0 \leqslant k<s$, and canonically isomorphic to $(A / I)^{\wedge s} \wedge B(I)[k-s+1]$, for $k \geqslant s$. Under this isomorphism, the face maps become $d_{r}=(A / I)^{\wedge s} \wedge d_{i}$, if $r=s-1+i$ with $0<i<k-s+1$, and $d_{r}=*$, otherwise. The desired isomorphism

$$
\alpha:(A / I)^{\wedge s} \wedge B(I) \wedge \Delta^{s-1} / \partial \Delta^{s-1} \stackrel{\sim}{\rightarrow} \operatorname{gr}_{s} \operatorname{TH}(A, I)_{0}^{\dagger}
$$

is then induced by the maps of symmetric spectra

$$
\alpha_{k}:(A / I)^{\wedge s} \wedge B(I)[k-s+1] \wedge \Delta_{+}^{k-s+1} \wedge \Delta_{+}^{s-1} \rightarrow \operatorname{gr}_{s} \operatorname{TH}(A, I)_{0}^{\dagger}[k] \wedge \Delta_{+}^{k}
$$


that, in turn, are induced by the maps of pointed spaces

$$
\alpha_{k}^{\prime}: \Delta_{+}^{k-s+1} \wedge \Delta_{+}^{s-1} \rightarrow \Delta_{+}^{k}
$$

defined by the formula

$$
\alpha_{k}^{\prime}\left(\left(x_{0}, \ldots, x_{k-s+1}\right),\left(y_{0}, \ldots, y_{s}\right)\right)=\left(y_{1}, \ldots, y_{s}, y_{0} x_{0}, \ldots, y_{0} x_{k-s+1}\right) .
$$

One checks that the maps $\alpha_{k}, k \geqslant 0$, factor through the equivalence relation that defines the realization and induce the isomorphism $\alpha$ as stated.

The proof of the second isomorphism is similar but notationally more involved. The symmetric spectrum $\operatorname{gr}_{u}^{\prime} \operatorname{gr}_{s} \operatorname{TH}(A, I)_{1}^{\dagger}[k]$ for $0 \leqslant k<s$, is trivial, and for $k \geqslant s$, is canonically isomorphic to the wedge sum

$$
\bigvee(A / I)^{\wedge n_{0}} \wedge B(I)\left[k_{1}\right] \wedge(A / I)^{\wedge n_{1}} \wedge \cdots \wedge B(I)\left[k_{\ell}\right] \wedge(A / I)^{\wedge n_{\ell}} \wedge I^{\wedge u}
$$

that ranges over all pairs of sequences of non-negative integers $\left(n_{0}, \ldots, n_{\ell}\right)$ and $\left(k_{1}, \ldots, k_{\ell}\right)$ such that $\ell \geqslant 1$, such that $n_{i}>0$, for $i>0$, and such that $n_{0}+\cdots+n_{\ell}=$ $s$ and $k_{1}+\cdots+k_{\ell}=k-u-s+1$. The face map $d_{r, s}$ takes the summand indexed by $\left(n_{0}, \ldots, n_{\ell}\right)$ and $\left(k_{1}, \ldots, k_{t}, \ldots, k_{\ell}\right)$ to the summand indexed by $\left(n_{0}, \ldots, n_{\ell}\right)$ and $\left(k_{1}, \ldots, k_{t}-1, \ldots, k_{\ell}\right)$ by the map induced from

$$
d_{i}: B(I)\left[k_{t}\right] \rightarrow B(I)\left[k_{t}-1\right],
$$

if there exists $1 \leqslant t \leqslant \ell$ and $0<i<k_{t}$ such that

$$
r=n_{0}+k_{1}+n_{1}+\cdots+k_{t-1}+n_{t-1}-1+i,
$$

and by the trivial map, otherwise. In particular, the face maps preserve the index $\left(n_{0}, \ldots, n_{\ell}\right)$. It follows that, as a semi-simplicial symmetric spectrum,

$$
\operatorname{gr}_{u}^{\prime} \operatorname{gr}_{s} \operatorname{TH}(A, I)_{1}^{\dagger}[-]=\bigvee E\left(n_{0}, \ldots, n_{\ell}\right)[-]
$$

where $E\left(n_{0}, \ldots, n_{\ell}\right)[-]$ consists of all the wedge summands above with the index $\left(n_{0}, \ldots, n_{\ell}\right)$ fixed. To finish the proof of the lemma, we observe that there is a natural isomorphism of symmetric spectra

$$
\beta: B(I)^{\wedge \ell} \wedge(A / I)^{\wedge s} \wedge I^{\wedge u} \wedge \Delta^{s+u-1} / \partial \Delta^{s+u-1} \stackrel{\sim}{\rightarrow}\left\|[k] \mapsto E\left(n_{0}, \ldots, n_{\ell}\right)[k]\right\| .
$$

The map $\beta$ is defined in a manner similar to the map $\alpha$ above and is induced from the maps of pointed spaces

$$
\beta_{\left(k_{1}, \ldots, k_{\ell}\right)}^{\prime}: \Delta_{+}^{k_{1}} \wedge \cdots \wedge \Delta_{+}^{k_{\ell}} \wedge \Delta_{+}^{s+u-1} \rightarrow \Delta_{+}^{k}
$$

that to the $(\ell+1)$-tuple of points with barycentric coordinates

$$
\left(\left(x_{1,0}, \ldots, x_{1, k_{1}}\right), \ldots,\left(x_{\ell, 0}, \ldots, x_{\ell, k_{\ell}}\right),\left(y_{0}, \ldots, y_{s+u-1}\right)\right)
$$

associates the point with barycentric coordinates

$$
\begin{gathered}
\left(y_{1}, \ldots, y_{n_{0}}, y_{0}^{\prime} x_{1,0}, \ldots, y_{0}^{\prime} x_{1, k_{1}}, y_{n_{0}+1}, \ldots, y_{n_{0}+n_{1}}, \ldots\right. \\
\left.\ldots, y_{0}^{\prime} x_{\ell, 0}, \ldots, y_{0}^{\prime} x_{\ell, k_{\ell}}, y_{n_{0}+\cdots+n_{\ell-1}+1}, \ldots, y_{s+u-1}\right)
\end{gathered}
$$

where $y_{0}^{\prime}=y_{0} / \ell$. This completes the proof of the lemma.

We wish to evaluate the spectrum homology

$$
H_{q}\left(B(I), \mathbb{F}_{p}\right)=\pi_{q}\left(B(I) \wedge \mathbb{F}_{p}\right) .
$$

The absence of a unit in $I$ and the resulting absence of degeneracy maps in $B(I)[-]$ makes this a non-trivial problem. To wit, the Eilenberg-Zilber theorem, which 
states that the total complex and the diagonal complex associated with a bisimplicial abelian group are chain homotopy equivalent, is not valid in the absence of degeneracy maps. Following Wodzicki [30 Thm. 9.5] we prove the following result:

LEMMA 2.8. Let I be a non-unital associative ring, let p be a prime, and suppose that the pro-abelian group

$$
\left\{\operatorname{Tor}_{q}^{\mathbb{Z} \ltimes I^{m}}(\mathbb{Z}, \mathbb{Z} / p)\right\}_{m \geqslant 1}
$$

is zero, for all positive integers $q$. Then the pro-abelian group

$$
\left\{H_{q}\left(B\left(I^{m}\right), \mathbb{Z} / p\right)\right\}_{m \geqslant 1}
$$

is zero, for all integers $q$.

PROOF. We first recall the algebraic bar-construction. Let $\Lambda$ be a commutative ring, and let $J$ be a non-unital associative $\Lambda$-algebra. The algebraic bar-construction of $J$ is the semi-simplicial $\Lambda$-module $B^{\Lambda}(J)[-]$ that in degree zero is 0 , in positive degree $k$ is the $k$-fold tensor product of copies of $J$, and whose face maps are given by

$$
d_{r}\left(a_{1} \otimes \cdots \otimes a_{k}\right)= \begin{cases}a_{1} \otimes \cdots \otimes a_{r} a_{r+1} \otimes \cdots \otimes a_{k} & 0<r<k \\ 0 & r=0 \text { or } r=k\end{cases}
$$

We write $B_{*}^{\Lambda}(J)$ for the associative chain complex of $\Lambda$-modules, where the differential is given by the alternating sum of the face maps.

We show that in the skeleton spectral sequence

$$
E_{s, t}^{1}=\left\{H_{t}\left(B\left(I^{m}\right)[s], \mathbb{F}_{p}\right)\right\} \Rightarrow\left\{H_{s+t}\left(B\left(I^{m}\right), \mathbb{F}_{p}\right)\right\},
$$

the pro-abelian groups $E_{s, t}^{2}$ are zero, for all integers $s$ and $t$. This implies that also the pro-abelian groups $E_{s, t}^{\infty}$ are zero, for all integers $s$ and $t$. Since the induced filtration of the pro-abelian groups in the abutment is finite, the statement follows. The Künneth formula shows that there is a canonical isomorphism of complexes of graded pro-abelian groups

$$
\left\{B_{*}^{\mathbb{F}_{p}}\left(H_{*}\left(I^{m}, \mathbb{F}_{p}\right)\right)\right\} \stackrel{\sim}{\longrightarrow}\left\{H_{*}\left(B\left(I^{m}\right)[-], \mathbb{F}_{p}\right)\right\}=E_{*, *}^{1} .
$$

Suppose first that the additive group of the $\operatorname{ring} I$ is torsion free. Then the canonical map of graded $\mathbb{F}_{p}$-algebras

$$
I^{m} \otimes H_{*}\left(\mathbb{Z}, \mathbb{F}_{p}\right) \rightarrow H_{*}\left(I^{m}, \mathbb{F}_{p}\right)
$$

is an isomorphism. Hence, we must show that the graded pro-abelian group

$$
\left\{H_{s}\left(B_{*}^{\mathbb{F} p}\left(I^{m} \otimes H_{*}\left(\mathbb{Z}, \mathbb{F}_{p}\right)\right)\right)\right\}
$$

is zero, for all integers $s$. Here we appeal to (the proof of) [30 Thm. 9.5]: Since $H_{*}\left(\mathbb{Z}, \mathbb{F}_{p}\right)$ is unital, it suffices to prove that the pro-abelian group

$$
\left\{H_{s}\left(B_{*}^{\mathbb{F}_{p}}\left(I^{m} \otimes \mathbb{F}_{p}\right)\right)\right\}
$$

is zero, for all integers $s$. For $s \leqslant 0$, this pro-abelian group is zero, by definition. For $s>0$, the canonical maps of pro-abelian groups

$$
\left\{\operatorname{Tor}_{s}^{\mathbb{Z} \ltimes I^{m}}\left(\mathbb{Z}, \mathbb{F}_{p}\right)\right\} \rightarrow\left\{H_{s}\left(B_{*}^{\mathbb{Z}}\left(I^{m}\right) \otimes \mathbb{F}_{p}\right)\right\} \rightarrow\left\{H_{s}\left(B_{*}^{\mathbb{F}_{p}}\left(I^{m} \otimes \mathbb{F}_{p}\right)\right)\right\}
$$

are isomorphisms, since $I$ is torsion free [25. Lemma 1.1], and the pro-abelian group on the left-hand side is zero by assumption. This completes the proof for $I$ torsion free. The general case is proved in a similar manner by first functorially replacing 
the non-unital associative $I^{m}$ by a simplicial non-unital associative ring $F\left(I^{m}\right)[-]$ such that for all $s \geqslant 0$, the non-unital associative $\operatorname{ring} F\left(I^{m}\right)[s]$ is torsion free as an abelian group.

Proof of Thm. 2.2 We prove that the canonical map

$$
\left\{\mathrm{TH}_{q}\left(I^{m}, \mathbb{Z} / p\right)\right\}_{m \geqslant 1} \rightarrow\left\{\mathrm{TH}_{q}\left(A, I^{m}, \mathbb{Z} / p\right)\right\}_{m \geqslant 1}
$$

is an isomorphism of pro-abelian groups, for all integers $q$. It suffices by 23 , Cor. 5.8] to show that the canonical map

$$
\left\{H_{q}\left(\mathrm{TH}\left(I^{m}\right), \mathbb{Z} / p\right)\right\}_{m \geqslant 1} \rightarrow\left\{H_{q}\left(\mathrm{TH}\left(A, I^{m}\right), \mathbb{Z} / p\right)\right\}_{m \geqslant 1}
$$

is an isomorphism of pro-abelian groups, for all integers $q$. This map is the edgehomomorphism in the spectral sequence of pro-abelian groups

$$
E_{s, t}^{1}=\left\{H_{t}\left(\operatorname{gr}_{s} \mathrm{TH}\left(A, I^{m}\right), \mathbb{Z} / p\right)\right\} \Rightarrow\left\{H_{s+t}\left(\mathrm{TH}\left(A, I^{m}\right), \mathbb{Z} / p\right)\right\} .
$$

Hence, it suffices to show that for all $s>0$, the pro-abelian groups $E_{s, t}^{1}$ are zero. We have a direct sum decomposition

$$
E_{s, t}^{1}=H_{t}\left(\operatorname{gr}_{s} \operatorname{TH}\left(A, I^{m}\right)_{0}^{\dagger}, \mathbb{Z} / p\right) \oplus H_{t}\left(\operatorname{gr}_{s} \operatorname{TH}\left(A, I^{m}\right)_{1}^{\dagger}, \mathbb{Z} / p\right)
$$

and an additional first-quadrant spectral sequence

$$
{ }^{\prime} E_{u, v}^{1}=\left\{H_{v}\left(\operatorname{gr}_{u}^{\prime} \operatorname{gr}_{s} \operatorname{TH}\left(A, I^{m}\right)_{1}^{\dagger}, \mathbb{Z} / p\right)\right\} \Rightarrow\left\{H_{u+v}\left(\operatorname{gr}_{s} \operatorname{TH}\left(A, I^{m}\right)_{1}^{\dagger}, \mathbb{Z} / p\right) .\right.
$$

It follows that to prove the theorem, it will suffice to show that the pro-abelian groups $\left\{H_{q}\left(\operatorname{gr}_{s} \operatorname{TH}\left(A, I^{m}\right), \mathbb{Z} / p\right)_{0}^{\dagger}\right\}$ and $\left\{H_{q}\left(\operatorname{gr}_{u}^{\prime} \operatorname{gr}_{s} \operatorname{TH}\left(A, I^{m}\right), \mathbb{Z} / p\right)_{1}^{\dagger}\right\}$ are zero, for all positive integers $s$ and all non-negative integers $u$ and $q$. But this is an immediate consequence of Lemmas 2.7 and 2.8

\section{Proof of Theorems 1, 2, and 4}

In this section we complete the proof of Thm. 1 1 and prove Thms. 2 and 4 The following result is similar to the proof of [7, Thm. 5.3]:

Lemma 3.1. Suppose that Thm. 回holds for every triple $(A, B, I)$, where I can be embedded as a two-sided ideal of a free unital associative ring. Then Thm. 1 holds for all triples $(A, B, I)$.

Proof. Let $X$ be a functor from unital associative rings to symmetric spectra that satisfies that for every pair of rings $(A, B)$ the canonical map

$$
X(A \times B) \rightarrow X(A) \times X(B)
$$

is a weak equivalence. We extend $X$ to a functor $X^{\prime}$ defined on non-unital associative rings as follows. If $I$ is a non-unital associative ring, we define $X^{\prime}(I)$ to be the homotopy fiber of the map $X(\mathbb{Z} \ltimes I) \rightarrow X(\mathbb{Z})$ induced by the canonical projection. Suppose that $A$ is a unital associative ring with unit $e$. Then the map $\mathbb{Z} \ltimes A \rightarrow \mathbb{Z} \times A$ that takes $(x, a)$ to $(x, x \cdot e+a)$ is a ring isomorphism, and hence, we obtain a weak equivalence

$$
X(\mathbb{Z} \ltimes A) \stackrel{\sim}{\rightarrow} X(\mathbb{Z} \times A) \stackrel{\sim}{\rightarrow} X(\mathbb{Z}) \times X(A)
$$

of symmetric spectra over $X(\mathbb{Z})$. These maps induce a weak equivalence of the homotopy fiber $X^{\prime}(A)$ of the map $X(\mathbb{Z} \ltimes A) \rightarrow X(\mathbb{Z})$ and the homotopy fiber 
$X^{\prime \prime}(A)$ of the canonical projection from $X(\mathbb{Z}) \times X(A)$ to $X(\mathbb{Z})$. This shows that we have natural weak equivalences

$$
X^{\prime}(A) \stackrel{\sim}{\rightarrow} X^{\prime \prime}(A) \stackrel{\sim}{\leftarrow} X(A) .
$$

Hence, the functor $X^{\prime}$ extends, up to canonical weak equivalence, the functor $X$ to non-unital associative rings. In the following, we will write $X$ instead of $X^{\prime}$ for the extended functor.

Let $X^{n}(A)$ be the homotopy fiber of the cyclotomic trace map

$$
K(A) \rightarrow \mathrm{TC}^{n}(A ; p) .
$$

Then Thm. 1 is equivalent to the statement that for every unital associative ring $A$ and two-sided ideal $I \subset A$, for all integers $q$, all primes $p$, and all positive integers $v$, the canonical map

$$
\left\{X_{q}^{n}\left(I, \mathbb{Z} / p^{v}\right)\right\}_{n \geqslant 1} \rightarrow\left\{X_{q}^{n}\left(A, I, \mathbb{Z} / p^{v}\right)\right\}_{n \geqslant 1}
$$

is an isomorphism of pro-abelian groups. We let $F(A)$ denote the free unital associative ring generated by the underlying set of $A$. There is a canonical surjective ring homomorphism $F(A) \rightarrow A$. We consider the following diagram of non-unital associative rings, where the rows and columns of the underlying diagram of abelian groups all are short-exact sequences:

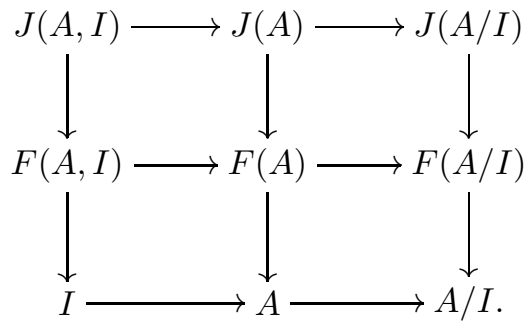

Since the non-unital rings in the top row can be embedded as ideals of free unital associative rings, the diagram, by hypothesis, gives rise to the following map of long-exact sequences of pro-abelian groups:

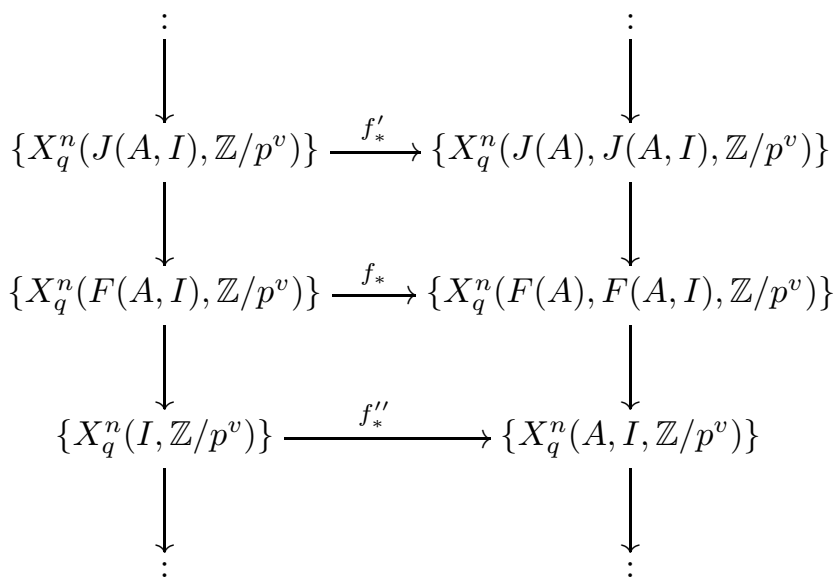

Finally, since the non-unital associative rings $J(A, I)$ and $F(A, I)$ can be embedded in a free unital associative ring, the maps labelled $f_{*}^{\prime}$ and $f_{*}$ are isomorphisms of pro-abelian groups. Hence, so is the map labelled $f_{*}^{\prime \prime}$. This completes the proof. 
We recall the following result of Cuntz and Quillen [7, Prop. 4.1]. Let $k$ be a field, and let $I \subset T$ be a two-sided ideal of a free unital associative $k$-algebra. Then there exists a $k$-linear section

$$
\alpha: I \rightarrow T \otimes_{k} I
$$

of the multiplication map $\mu: T \otimes_{k} I \rightarrow I$ such that the diagram

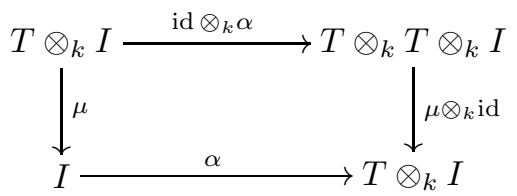

commutes. This is stated in loc.cit. for $I$ a two-sided ideal of free unital associative $\mathbb{C}$-algebra, but the proof works for every ground field. The diagram shows, in particular, that the map $\alpha$ takes $I^{2}$ to $I \otimes_{k} I$. We use this to prove the following result:

Proposition 3.3. Let $I \subset T$ be a two-sided ideal of a free unital associative ring. Then for all primes $p$, and all positive integer $v$ and $q$, the following proabelian group is zero:

$$
\left\{\operatorname{Tor}_{q}^{\mathbb{Z} \ltimes I^{m}}\left(\mathbb{Z}, \mathbb{Z} / p^{v}\right)\right\}_{m \geqslant 1} .
$$

Proof. Since $\mathbb{Z} \ltimes I^{m}$ is flat over $\mathbb{Z}$, the group $\operatorname{Tor}_{q}^{\mathbb{Z} \ltimes I^{m}}\left(\mathbb{Z}, \mathbb{Z} / p^{v}\right)$ is canonically isomorphic to the $q$ th homology group of the bar-complex $B_{*}^{\mathbb{Z}}\left(I^{m}\right) \otimes \mathbb{Z} / p^{v}$; see the proof of Lemma 2.8 Hence, it suffices to show that for every $m \geqslant 1$, there exists $m^{\prime} \geqslant m$ such that the canonical map

$$
B_{*}^{\mathbb{Z}}\left(I^{m^{\prime}}\right) \otimes \mathbb{Z} / p^{v} \rightarrow B_{*}^{\mathbb{Z}}\left(I^{m}\right) \otimes \mathbb{Z} / p^{v}
$$

induces the zero map on homology in positive degrees. Since also $I^{m} \subset T$ is a two-sided ideal of a free unital associative ring, it suffices to treat the case $m=1$. By simple induction, it also suffices to consider the case $v=1$.

The tensor-product $I \otimes \mathbb{F}_{p}$ is a non-unital associative $\mathbb{F}_{p}$-algebra, and there is a canonical isomorphism of complexes of $\mathbb{F}_{p}$-vector spaces

$$
B_{*}^{\mathbb{Z}}(I) \otimes \mathbb{F}_{p} \stackrel{\sim}{\rightarrow} B_{*}^{\mathbb{Z}}\left(I \otimes \mathbb{F}_{p}\right) .
$$

We let $\left(I \otimes \mathbb{F}_{p}\right)^{\prime}$ and $\overline{I \otimes \mathbb{F}_{p}}$ denote the kernel and image, respectively, of the map $I \otimes \mathbb{F}_{p} \rightarrow T \otimes \mathbb{F}_{p}$ induced by the inclusion. Then we have the following extension of non-unital associative $\mathbb{F}_{p}$-algebras:

$$
\left(I \otimes \mathbb{F}_{p}\right)^{\prime} \rightarrow I \otimes \mathbb{F}_{p} \rightarrow \overline{I \otimes \mathbb{F}_{p}} .
$$

The non-unital associative $\mathbb{F}_{p}$-algebra $\overline{I \otimes \mathbb{F}_{p}}$ is a two-sided ideal of the free unital associative $\mathbb{F}_{p}$-algebra $T \otimes \mathbb{F}_{p}$. Let

$$
\alpha: \overline{I \otimes \mathbb{F}_{p}} \rightarrow\left(T \otimes \mathbb{F}_{p}\right) \otimes\left(\overline{I \otimes \mathbb{F}_{p}}\right)
$$

be the section of the multiplication from (3.2). Then the formula

$$
s\left(x_{1} \otimes \cdots \otimes x_{n}\right)=(-1)^{n} x_{1} \otimes \cdots \otimes x_{n-1} \otimes \alpha\left(x_{n}\right)
$$

defines a null-homotopy of the chain map

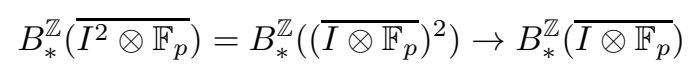

induced by the canonical inclusion. 
We next let $C_{*}\left(I \otimes \mathbb{F}_{p}\right)$ be the kernel complex of the chain-map

$$
B_{*}^{\mathbb{Z}}\left(I \otimes \mathbb{F}_{p}\right) \rightarrow B_{*}^{\mathbb{Z}}\left(\overline{I \otimes \mathbb{F}_{p}}\right)
$$

induced by the canonical projection. The $\mathbb{F}_{p}$-vector space $C_{n}\left(I \otimes \mathbb{F}_{p}\right)$ is generated by the tensors $x_{1} \otimes \cdots \otimes x_{n}$, where at least one factor is in $\left(I \otimes \mathbb{F}_{p}\right)^{\prime}$. We claim that the chain-map

$$
C_{*}\left(I^{2} \otimes \mathbb{F}_{p}\right) \rightarrow C_{*}\left(I \otimes \mathbb{F}_{p}\right)
$$

induced by the canonical inclusion is equal to zero. This follows immediately from the statement that the map induced by the canonical inclusion

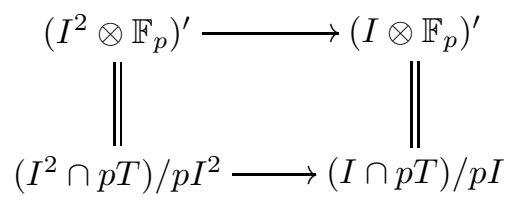

is zero. Hence, it suffices to show that $I^{2} \cap p T \subset p I$, or equivalently, that if $x \in I$ and $y \in I$, and if $x y \in p T$, then $x y \in p I$. Now, if $x y \in p T$, then $x \in p T$ or $y \in p T$, since a free unital associative $\mathbb{F}_{p}$-algebra does not have left or right zero-divisors $\mathbf{5}$, p. 32]. If $x \in p T$, we write $x=p x^{\prime}$ with $x^{\prime} \in T$. Then $x y=p x^{\prime} y$ and since $I$ is a left ideal, $x^{\prime} y \in I$. Similarly, if $y \in p T$, we write $y=p y^{\prime}$ with $y^{\prime} \in T$. Then $x y=p x y^{\prime}$, and since $I$ is a right ideal, $x y^{\prime} \in I$. In either case, $x y \in p I$ as desired.

Finally, a diagram chase shows that the chain map

$$
B_{*}^{\mathbb{Z}}\left(I^{4} \otimes \mathbb{F}_{p}\right) \rightarrow B_{*}^{\mathbb{Z}}\left(I \otimes \mathbb{F}_{p}\right)
$$

induces the zero map on homology in positive degrees.

Lemma 3.4. Let $f: A \rightarrow B$ be a map of unital associative rings and let $I \subset A$ be a two-sided ideal such that $f: I \rightarrow f(I)$ is an isomorphism onto an ideal of $B$. Let $p$ be a prime and let $v$ be a positive integer. Suppose that the cyclotomic trace induces an isomorphism of pro-abelian groups

$$
\left\{K_{q}\left(A, B, I^{m}, \mathbb{Z} / p^{v}\right)\right\}_{m \geqslant 1} \stackrel{\sim}{\rightarrow}\left\{\mathrm{TC}_{q}^{n}\left(A, B, I^{m} ; p, \mathbb{Z} / p^{v}\right)\right\}_{m, n \geqslant 1},
$$

for all integers $q$. Then the map induced by the cyclotomic trace

$$
K_{q}\left(A, B, I, \mathbb{Z} / p^{v}\right) \rightarrow\left\{\mathrm{TC}_{q}^{n}\left(A, B, I ; p, \mathbb{Z} / p^{v}\right)\right\}_{n \geqslant 1}
$$

is an isomorphism of pro-abelian groups, for all integers $q$.

Proof. We note that for all positive integers $m$ and $n$, we have a commutative diagram of spectra in which the columns are distinguished triangles.

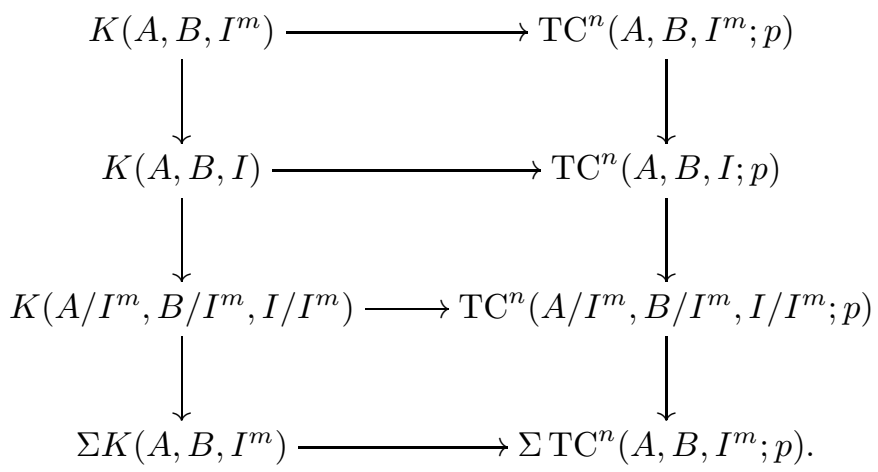

27 
The induced diagram of homotopy groups with $\mathbb{Z} / p^{v}$-coefficients gives rise, as $m$ and $n$ vary, to a map of long-exact sequences of pro-abelian groups. The map of pro-abelian groups induced by the top horizontal map in the diagram above is an isomorphism by assumption, and we wish to show that the map of proabelian groups induced by the second horizontal map in the diagram above is an isomorphism. By a five-lemma argument this is equivalent to showing that the map of pro-abelian groups induced from the third horizontal map in the diagram above is an isomorphism. Now, it follows from [21 and from [11, Thm. 2.2.1] that for $m \geqslant 1$ fixed, the cyclotomic trace induces an isomorphism of pro-abelian groups

$$
K_{q}\left(A / I^{m}, B / I^{m}, I / I^{m}, \mathbb{Z} / p^{v}\right) \stackrel{\sim}{\rightarrow}\left\{\mathrm{TC}_{q}^{n}\left(A / I^{m}, B / I^{m}, I / I^{m} ; p, \mathbb{Z} / p^{v}\right)\right\}_{n \geqslant 1} .
$$

But then the map of pro-abelian groups obtained by also allowing $m \geqslant 1$ to vary is an isomorphism. This completes the proof.

Proof of Thm. 1. By Lemma 3.1] we can assume that the non-unital associative ring $I$ can be embedded as a two-sided ideal $I \subset T$ of a free unital associative ring. Moreover, by Lemma 3.4 it suffices to show that for all integers $q$, all primes $p$, and all positive integers $v$, the cyclotomic trace induces an isomorphism of proabelian groups

$$
\left\{K_{q}\left(A, B, I^{m}, \mathbb{Z} / p^{v}\right)\right\}_{m \geqslant 1} \stackrel{\sim}{\rightarrow}\left\{\mathrm{TC}_{q}^{n}\left(A, B, I^{m} ; p, \mathbb{Z} / p^{v}\right)\right\}_{m, n \geqslant 1} .
$$

But Prop. 3.3 and Thms. 1.1 and 2.1 show that both pro-abelian groups are zero.

Finally, we prove Thms. 2 and 4 of the introduction.

Proof of Thm. 2. Let $X \rightarrow$ Spec $k$ be a curve over a field $k$, that is, a separated morphism of finite type with $X$ of dimension one. We wish to show that for all $q \geqslant r$, the cyclotomic trace

$$
K_{q}\left(X, \mathbb{Z} / p^{v}\right) \rightarrow\left\{\mathrm{TC}_{q}^{n}\left(X ; p, \mathbb{Z} / p^{v}\right)\right\}_{n \geqslant 1}
$$

is an isomorphism of pro-abelian groups. We first reduce to the case where $X$ is affine. A covering $\left\{U_{i}\right\}$ of $X$ by affine open subschemes gives rise to spectral sequences of pro-abelian groups

$$
E_{s, t}^{2}=\check{H}^{-s}\left(\left\{U_{i}\right\}, K_{t}\left(-, \mathbb{Z} / p^{v}\right)\right) \Rightarrow K_{s+t}\left(X, \mathbb{Z} / p^{v}\right)
$$

and

$$
E_{s, t}^{2}=\left\{\check{H}^{-s}\left(\left\{U_{i}\right\}, \mathrm{TC}_{t}^{n}\left(-; p, \mathbb{Z} / p^{v}\right)\right)\right\}_{n \geqslant 1} \Rightarrow\left\{\mathrm{TC}_{s+t}^{n}\left(X ; p, \mathbb{Z} / p^{v}\right)\right\}_{n \geqslant 1} .
$$

Indeed, for $K$-theory, this follows from [27. Thm. 10.3], and for topological cyclic homology, this follows directly from the definition of the topological cyclic homology of a scheme 10 Def. 3.2.3]. Assuming the theorem for affine schemes, the cyclotomic trace induces a map of spectral sequences which is an isomorphism on $E_{s, t}^{2}$ for $t \geqslant r$. But then the cyclotomic trace induces an isomorphism of the pro-abelian groups in the abutments in degrees $q \geqslant r$.

So assume that $X$ is affine. By an argument similar to the proof of Lemma 3.4 above, we can further assume that $X$ is reduced. By induction on the number of irreducible components in $X$, we can further reduce to the case where $X$ is irreducible. Indeed, let $Z$ and $Z^{\prime}$ be two proper closed subschemes of $X$ and suppose that $Z$ and $Z^{\prime}$ cover $X$, or equivalently, that the corresponding ideals $I$ and $I^{\prime}$ of 
the coordinate ring $A$ intersect trivially. Then it follows from Thm. 1 that the cyclotomic trace induces an isomorphism of pro-abelian groups

$$
K_{q}\left(A, A / I^{\prime}, I, \mathbb{Z} / p^{v}\right) \stackrel{\sim}{\longrightarrow}\left\{\mathrm{TC}_{q}^{n}\left(A, A / I^{\prime}, I ; p, \mathbb{Z} / p^{v}\right)\right\}_{n \geqslant 1},
$$

for all integers $q$. This proves the induction step.

Finally, let $X \rightarrow$ Spec $k$ be a reduced and irreducible affine curve. The coordinate ring $A$ is an integral domain, and we let $B$ be the integral closure. We claim that the conductor ideal $I=\{a \in A \mid a B \subset A\}$, that is the largest ideal of $A$ which is also an ideal of $B$, is non-zero. Indeed, the extension $A \subset B$ is finite, so we can write $B=b_{1} A+\cdots+b_{m} A$. Since $A$ and $B$ have the same quotient field, there are non-zero elements $a_{i} \in A$ such that $a_{i} b_{i} \in A, 1 \leqslant i \leqslant m$. But then the product $a_{1} \ldots a_{m}$ is in $I$ which therefore is non-zero as claimed. The $\operatorname{ring} B$ is a regular $k$-algebra, and since $A$ and $B$ are of dimension one, the quotients $A / I$ and $B / I$ are finite $k$-algebras. Hence, we know from [10 Thm. 4.2.2] and from 21] and 11, Thm. 2.2.1] that the cyclotomic trace induces an isomorphism of pro-abelian groups

$$
K_{q}\left(R, \mathbb{Z} / p^{v}\right) \stackrel{\sim}{\rightarrow}\left\{\mathrm{TC}_{q}^{n}\left(R ; p, \mathbb{Z} / p^{v}\right)\right\}_{n \geqslant 1},
$$

for $q \geqslant r$, if $R$ is one of $B, A / I$, and $B / I$. But then Thm. 1 shows that this map is an isomorphism, for $q \geqslant r$, also if $R=A$.

Proof of Thm. 4 The ideal $I=|G| \cdot \mathfrak{M}$ is a common ideal of $A=\mathbb{Z}[G]$ and $B=\mathfrak{M}$ 1 Cor. XI.1.2], and hence, we can apply Thm. 1 It follows that the exactness of the sequence in the statement of Thm. 4 is equivalent to the exactness of the corresponding sequence with $\mathbb{Z}[G]$ and $\mathfrak{M}$ replaced by $\mathbb{Z}[G] / I$ and $\mathfrak{M} / I$. The latter are both artinian rings whose quotient by the Jacobson radical is a semisimple $\mathbb{F}_{p}$-algebra. Hence, the exactness of the latter sequence follows from $1 \mathbf{1 6}$ Thm. 5.1(i)] and from [11, Thm. 2.2.1].

\section{References}

[1] H. Bass, Algebraic K-theory, W. A. Benjamin, Inc., New York-Amsterdam, 1968.

[2] A. J. Berrick and W. G. Dwyer, The spaces that define algebraic K-theory, Topology 39 (2000), 225-228.

[3] M. Bökstedt and I. Madsen, Topological cyclic homology of the integers, K-theory (Strasbourg, 1992), Astérisque, vol. 226, 1994, pp. 57-143.

[4] A. K. Bousfield and D. M. Kan, Homotopy limits, completions and localizations, Lecture Notes in Math., vol. 304, Springer-Verlag, New York, 1972.

[5] P. M. Cohn, Free rings and their relations, London Mathematical Society Monographs, vol. 2, Academic Press, London-New York, 1971.

[6] G. Cortiñas, The obstruction to excision in K-theory and in cyclic homology, Invent. Math. 164 (2006), 143-173.

[7] J. Cuntz and D. Quillen, Excision in bivariant periodic cyclic cohomology, Invent. Math. 127 (1997), 67-98.

[8] R. K. Dennis and M. I. Krusemeyer, $K_{2}(A[X, Y] /(X Y))$, a problem of Swan, and related computations, J. Pure Appl. Alg. 15 (1979), 125-148.

[9] B. I. Dundas and H. Kittang, Excision for K-theory of connective ring spectra, Preprint 2005.

[10] T. Geisser and L. Hesselholt, Topological cyclic homology of schemes, K-theory (Seattle, 1997), Proc. Symp. Pure Math., vol. 67, 1999, pp. 41-87.

[11] _ On the K-theory and topological cyclic homology of smooth schemes over a discrete valuation ring, Trans. Amer. Math. Soc 358 (2006), 131-145.

[12] S. Geller, L. Reid, and C. A. Weibel, The cyclic homology and K-theory of curves, Bull. Amer. Math. Soc. 15 (1986), no. 2, 205-208.

[13] _ The cyclic homology and K-theory of curves, J. reine angew. Math. 393 (1989), 39-90. 
[14] L. Hesselholt, On the K-theory of the coordinate axes in the plane, Nagoya Math. J. (to appear).

[15] $\ldots$-theory of truncated polynomial algebras, Handbook of $K$-theory, Springer-Verlag, New York, 2005, pp. 71-110.

[16] L. Hesselholt and I. Madsen, On the K-theory of finite algebras over Witt vectors of perfect fields, Topology 36 (1997), 29-102.

[17] On the K-theory of nilpotent endomorphisms, Homotopy methods in algebraic topology (Boulder, CO, 1999), Contemp. Math., vol. 271, Amer. Math. Soc., Providence, RI, 2001, pp. 127-140.

[18] On the K-theory of local fields, Ann. of Math. 158 (2003), 1-113.

[19] P. S. Hirschhorn, Model categories and their localizations, Mathematical Surveys and Monographs, vol. 99, American Mathematical Society, Providence, RI, 2003.

[20] M. Hovey, B. Shipley, and J. Smith, Symmetric spectra, J. Amer. Math. Soc. 13 (2000), 149-208.

[21] R. McCarthy, Relative algebraic K-theory and topological cyclic homology, Acta Math. 179 (1997), 197-222.

[22] R. Oliver, Whitehead groups of finite groups, London Mathematical Society Lecture Note Series, vol. 132, Cambridge University Press, Cambridge, 1988.

[23] I. A. Panin, On a theorem of Hurewicz and $K$-theory of complete discrete valuation rings, Math. USSR Izvestiya 29 (1987), 119-131.

[24] B. Shipley, Symmetric ring spectra and topological Hochschild homology, K-theory 19 (2000), $155-183$.

[25] A. A. Suslin, Excision in the integral algebraic K-theory, Proc. Steklov Inst. Math. 208 (1995), 255-279.

[26] A. A. Suslin and M. Wodzicki, Excision in algebraic K-theory, Ann. of Math. 136 (1992), $51-122$.

[27] R. W. Thomason and T. Trobaugh, Higher algebraic K-theory of schemes and of derived categories, Grothendieck Festschrift, Volume III, Progress in Mathematics, vol. 88, 1990, pp. 247-435.

[28] C. A. Weibel, Mayer-Vietoris sequences and $\bmod p$ K-theory, Algebraic $K$-theory, Part I (Oberwolfach, 1980), Lecture Notes in Math., vol. 966, Springer-Verlag, New York, 1982.

[29] An introduction to homological algebra, Cambridge Studies in Advanced Mathematics, vol. 38, Cambridge University Press, Cambridge, 1994.

[30] M. Wodzicki, Excision in cyclic homology and in rational algebraic K-theory, Ann. of Math. 129 (1989), 591-639.

University of Southern California, los Angeles, California

E-mail address: geisser@math.usc.edu

Massachusetts Institute of Technology, Cambridge, Massachusetts

E-mail address: larsh@math.mit.edu 